Document downloaded from:

http://hdl.handle.net/10251/40252

This paper must be cited as:

Hernández-Orallo, J. (2013). ROC curves for regression. Pattern Recognition. 46(12):33953411. doi:10.1016/j.patcog.2013.06.014.

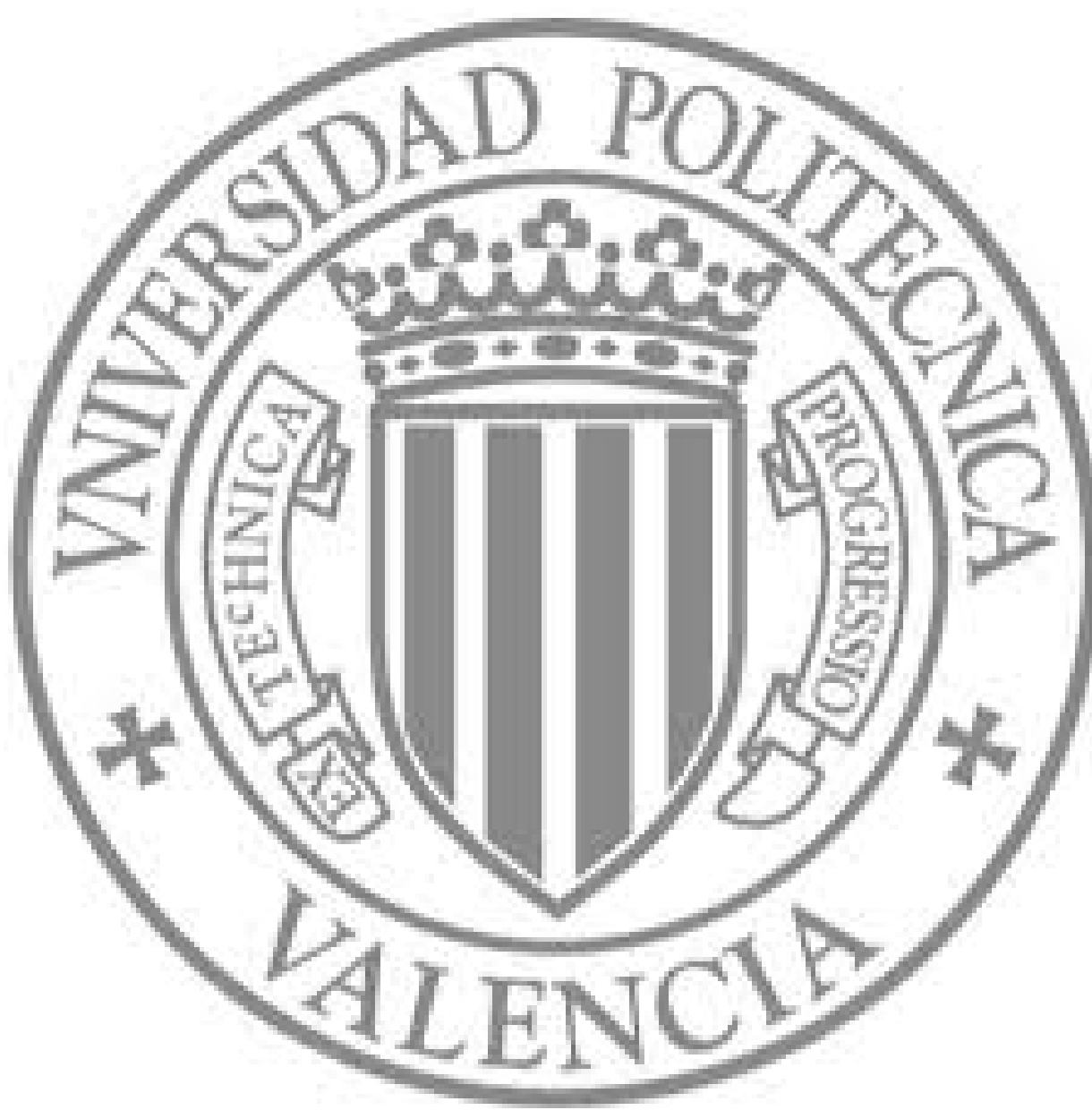

The final publication is available at

http://dx.doi.org/10.1016/j.patcog.2013.06.014

Copyright Elsevier 


\title{
ROC Curves for Regression
}

\author{
José Hernández-Orallo ${ }^{\mathrm{a}}$ \\ ${ }^{a}$ Departament de Sistemes Informàtics i Computació, Universitat Politècnica de València \\ Camí de Vera s/n, E-46022, València, Spain. Tel: +34963877007, Fax: +34963877359
}

\begin{abstract}
Receiver Operating Characteristic (ROC) analysis is one of the most popular tools for the visual assessment and understanding of classifier performance. In this paper we present a new representation of regression models in the so-called regression ROC (RROC) space. The basic idea is to represent over-estimation against under-estimation. The curves are just drawn by adjusting a shift, a constant that is added (or subtracted) to the predictions, and plays a similar role as a threshold in classification. From here, we develop the notions of optimal operating condition, convexity, dominance, and explore several evaluation metrics that can be shown graphically, such as the area over the RROC curve (AOC). In particular, we show a novel and significant result: the $A O C$ is equivalent to the error variance. We illustrate the application of RROC curves to resource estimation, namely the estimation of software project effort.
\end{abstract}

Keywords:

ROC Curves, Cost-sensitive regression, Operating condition, Asymmetric loss, Error variance, MSE decomposition.

\section{Introduction}

Receiver Operating Characteristic (ROC) analysis [36, 47, 4, 48, 19, 35, 14, $34]$ is a very popular technique for the graphical analysis of classification models. ROC analysis is profusely used in many areas [21, 37, 30, 31]: radiology, medicine, statistics, bioinformatics, machine learning, pattern recognition, etc. Also, some metrics derived from the ROC curve, such as the Area Under the ROC Curve (AUC), are now key for the evaluation and construction of classifiers $[15,38,50,42,32]$.

In classification, the traditional notion of operating condition is common and well understood. Classifiers may be trained for one cost proportion and class dis-

Email address: jorallo@dsic.upv.es (José Hernández-Orallo) 
tribution (both making the operating condition) and then deployed on a different operating condition. ROC space decomposes the performance of a classifier in a dual way. On the $x$-axis we show the false positive rate (FPR) and on the the $y$-axis we show the true positive rate (TPR). ROC curves neatly visualise how the TPR and the FPR change for different (crisp) classifiers or evolve for the same (soft) classifier (or ranker) for a range of thresholds. The notion of threshold is the fundamental idea to adapt a soft classifier to an operating condition. ROC analysis is the tool that illustrates how classifiers and threshold choices perform.

The adaptation of ROC analysis for regression has been attempted on many occasions. However, there is no such a thing as the 'canonical' adaptation of ROC analysis in regression, since regression and classification are different tasks, and the notion of operating condition may be completely different. In fact, the mere extension of ROC analysis to more than two classes has always been difficult because the degrees of freedom grow quadratically with the number of classes (see, e.g., $[46,17,44])$. Consequently it is even questionable whether a similar graphical representation of ROC curves in regression (or other tasks [26]) can even be figured out. Notable efforts towards ROC curves (or graphical tools) for regression are the Regression Error Characteristic (REC) Curves [3], the Regression Error Characteristic Surfaces (RECS) [51], the notion of utility-based regression [41] and the definition of ranking measures [43]. These approaches are based on gauging the tolerance, rejection rules or confidence levels. Some of these approaches actually convert the evaluation of a regression problem into a classification problem (tolerable estimation vs. intolerable estimation). However, none of these previous approaches started from a notion of 'operating condition', related to an asymmetric loss function. Also, the notion of threshold was not replaced by a similar concept playing its role for adjusting to the operating condition, and the dual positive-negative character in ROC analysis was blurred.

In this paper we present a graphical representation of regression performance based on a very usual view of operating condition. Many regression applications have deployment contexts where over-estimations are not equally costly as underestimations (or vice versa). This is called the loss asymmetry. Certainly, loss asymmetry is just one possible kind of operating condition (or one of its constituents), but it is a very common and important one in many applications.

The ROC space for regression (RROC space) is then defined by placing the total over-estimation on the $x$-axis and the total under-estimation on the $y$-axis. This duality leads to regions and isometrics in the ROC space where over-estimations have less cost than under-estimations and vice versa. There we can plot different regression models to see the notions of dominance. We also consider the construc- 
tion of hybrid regression models by 'interpolating' between points in the RROC space. Moreover, the plot leads to curves (called RROC curves) when we use the notion of shift, which is just a constant that we can add (or subtract) to example predictions in order to adjust the model to an asymmetric operating condition. This notion is parallel to the notion of threshold in classification. Interestingly, while we can derive the best shift for a dataset given an existing model (which boils down to finding the shift that makes its average error equal to zero if the cost is symmetric), there are some effective methods to determine this shift for the deployment data given an operating condition, as has been recently explored by [1][55]. All this leads to a more meaningful interpretation of what the ROC curves for regression really mean, and what their areas represent.

The paper is organised as follows. Section 2 introduces some notation, the problem of cost-sensitive evaluation and the use of asymmetric costs in regression. The RROC space is defined in section 3, where we represent several regression models as points, derive the isometrics of the space and develop the notions of hybrid models, dominance and convex hull. Section 4 introduces RROC curves, which are drawn by ranging a constant additive shift. We define an algorithm for plotting them and determine some of its properties in terms of segment slopes and convexity. Section 5 analyses the area over the RROC curve $(A O C)$, proving its linear relation to error variance, and showing that the squared error decomposition can be shown in RROC space. A real example is included in Section 6, which illustrates how RROC curves are used from training to deployment. Finally, section 7 closes the paper with an enumeration of issues for future investigation.

\section{Background}

In this section we introduce some notation and the basic concepts about costsensitive regression and the need of asymmetric loss functions.

\subsection{Notation}

Let us consider a multivariate input domain $\mathbb{X}$ and a univariate output domain $\mathbb{Y} \subset \mathbb{R}$. The domain space $\mathbb{D}$ is then $\mathbb{X} \times \mathbb{Y}$. Examples or instances are just pairs $\langle x, y\rangle \in \mathbb{D}$, and datasets are subsets (actually multi-sets) of $\mathbb{D}$. The length of a dataset will usually be denoted by $n$. A crisp regression model $m$ is a function $m: \mathbb{X} \rightarrow \mathbb{Y}$. When the regression model is crisp, we just represent the true value by $y$ and the estimated value by $\hat{y}$. Subindices will be used when referring to more than one example in a dataset. Vectors (unidimensional arrays) are denoted in boldface and its elements with subindices, e.g., $\mathbf{v}=\left(v_{1}, v_{2}, \ldots, v_{n}\right)$. Operations 
mixing arrays and scalar values will be allowed, specially in algorithms, as usual in the matrix arithmetic of many statistical computing languages. For instance, $\mathbf{v}+c$ means that the constant $c$ is added to all the elements in the vector $\mathbf{v}$. The mean of a vector is denoted by $\mu(\mathbf{v})$ and its standard deviation as $\sigma(\mathbf{v})$-over the population, i.e., divided by $n$. Given a dataset with $n$ instances $i=1 \ldots n$, the error vector $\mathbf{e}$ is defined such that $e_{i} \triangleq \hat{y}_{i}-y_{i}$. The value $\mu\left(\mathbf{e}^{2}\right)$ is known as the mean squared error $(M S E), \mu(\mathbf{e})$ is known as the mean error (or mean error bias, $M E B), \mu(|\mathbf{e}|)$ is known as the mean absolute error $(M A E)$ and $\sigma(\mathbf{e})^{2}$ as the error variance. Occasionally, we will drop the preceding $M$, especially when referring to total squared error $(S E)$, total error bias $(E B)$ and total absolute error $(A E)$.

\subsection{Cost-sensitive problems and loss functions}

In cost-sensitive learning [13], there are several features which describe a context, such as the data distribution, the costs of using some input variables and the loss of the errors over the output variables [52]. In this paper, we focus on loss functions over the output variable, which is the kind of costs ROC analysis deals with (typically integrated, along with the class distribution, within the notion of skew). A loss function is defined as follows:

Definition 1. A loss function is any function $\ell: \mathbb{Y} \times \mathbb{Y} \rightarrow \mathbb{R}$ which compares elements in the output domain. For convenience, the first argument will be the estimated value, and the second argument the actual value.

Typical examples of loss functions are the absolute error $\left(\ell^{A}\right)$ and the squared error $\left(\ell^{S}\right)$, with $\ell^{A}(\hat{y}, y) \triangleq|\hat{y}-y|$ and $\ell^{S}(\hat{y}, y) \triangleq(\hat{y}-y)^{2}$. These two loss functions are symmetric, i.e. for every $y$ and $r$ we have that $\ell(y+r, y)=\ell(y-r, y)$. Two of the most common metrics for evaluating regression, the mean absolute error (MAE) and the mean squared error (MSE) are derived from these losses.

\subsection{Asymmetric costs}

Actually, although symmetric loss functions (and derived metrics) are common for the evaluation of regression models, it is rarely the case that a real problem has a symmetric cost. For instance, the prediction of sales, consumptions, calls, prices, demands, survival times, positions, reliabilities, etc., rarely has a symmetric loss. For instance, a retailing company may need to predict how many items will be sold next week for stock (inventory) management purposes, e.g., in order to calculate how many items must be ordered. Depending on the kind of product, it is usually not the same to over-estimate (increasing stocking costs) 
than to under-estimate (an item goes out of stock and it cannot be sold or sold with delays). In fact, it is also rare to find applications where even an asymmetric cost is invariable. In the above-mentioned example, depending on the warehouse saturation, the cost (and the asymmetry) may change in a weekly or daily fashion. Because of this, a specialised model for each fixed given asymmetry is not a practical solution in general. This motivates the adaptation (or reframing) of models, rather than their re-training for each new asymmetric loss. This variability of the operating condition is at the core of ROC analysis in classification.

There has been an extensive amount of work on regression using asymmetric loss functions. In some cases, the loss function is embedded in the learning algorithm (see, e.g., [10, 45, 11]), which is useful if the operating condition is stable and we know it during training. However, the adaptation (or reframing) of an existing model to a different operating condition has also been investigated for regression (e.g., Granger [22, 23]). Many different kinds of asymmetric functions have been explored: Lin-Lin (asymmetric linear), Quad-Quad (asymmetric quadratic), Lin-Exp (approximately linear on one side and exponential on the other side) and Quad-Exp (approximately quadratic on one side and exponential on the other side) $[53,54,6,7,8,2,49]$. Some of these approaches try to adapt to the operating condition using complex (generally non-parametric) density functions, which is problematic for complex asymmetric loss functions.

As mentioned above, there are many possible asymmetric loss functions. The simplest (and perhaps most common) one is the asymmetric absolute error $\ell_{\alpha}^{A}$ :

Definition 2. The asymmetric absolute error $\ell_{\alpha}^{A}$, also known as Lin-Lin, is a loss function defined as follows:

$$
\ell_{\alpha}^{A}(\hat{y}, y) \triangleq \begin{cases}2 \alpha(y-\hat{y}) & \text { if } \hat{y}<y \\ 2(1-\alpha)(\hat{y}-y) & \text { otherwise }\end{cases}
$$

with $\alpha$ being the cost proportion (or asymmetry) between 0 and 1, with increasing values meaning higher cost for low predictions (under-estimation). In other words, when $\alpha=0$ we mean that predictions below the actual value have no cost. When $\alpha=1$ we mean that predictions above the actual value have no cost. When $\alpha=0.5$ we mean that costs above and below are symmetric.

\section{The RROC space}

For every regression model and dataset we can determine the error $e_{i}$ for each instance $i$ and summarise positive and negative errors separately. 
Definition 3. The total over-estimation is given by $O V E R \triangleq \sum_{i}\left\{e_{i} \mid e_{i}>0\right\}$ and the total under-estimation is given by UNDER $\triangleq \sum_{i}\left\{e_{i} \mid e_{i}<0\right\}$.

The following example illustrates this:

Example 1. Consider a regression model $m_{1}$ which is applied to a dataset with $n=10$ instances, producing the predicted values $\hat{y}$ that, compared with the actual values $y$, leads to the error values $e=\hat{y}-y$ :

\begin{tabular}{c|cccccccccc} 
& 1 & 2 & 3 & 4 & 5 & 6 & 7 & 8 & 9 & 10 \\
$\hat{y}$ & -0.082 & 3.323 & 2.320 & 1.080 & 7.893 & 4.983 & 5.121 & 3.442 & 2.083 & 1.112 \\
$y$ & 0.211 & 2.725 & 1.933 & 3.242 & 7.858 & 6.061 & 7.173 & 3.082 & 0.894 & 1.203 \\
$e$ & -0.293 & 0.598 & 0.387 & -2.162 & 0.035 & -1.078 & -2.052 & 0.360 & 1.189 & -0.091
\end{tabular}

The error row (e) shows the difference, which is positive for over-estimations and negative for under-estimations. The sum of over-estimations (OVER) is 2.569 while the sum of under-estimations (UNDER) is -5.676 . This regression model clearly under-estimates (it has a negative error bias, since $\mu(\mathbf{e})=-0.3107<0$ ). The MAE (0.825) and the MSE (1.219) do not show the asymmetry of predictions.

\subsection{Showing models in RROC space}

The basic idea of the ROC space for regression is to show model asymmetry:

Definition 4. The Regression Receiver Operating Characteristic (RROC) space is defined as a plot where we depict total over-estimation (OVER) on the x-axis and total under-estimation (UNDER) on the y-axis. Since OVER is always positive (but unbounded) and UNDER is always negative (but unbounded), we will typically place the point $(0,0)$ on the upper left corner (the RROC heaven), and will clip both the $x$-axis and $y$-axis as necessary to show the region of interest.

Figure 1 (left) shows the RROC space and the regression model $m_{1}$ in example 1. We will occasionally draw a diagonal dashed line $O V E R+U N D E R=0$ to show the points where the under-estimation equals the over-estimation.

Example 2. Consider a regression model $m_{2}$ which is applied to the same dataset as example 1:

\begin{tabular}{c|cccccccccc} 
& 1 & 2 & 3 & 4 & 5 & 6 & 7 & 8 & 9 & 10 \\
$\hat{y}$ & 0.786 & 2.078 & 0.587 & 1.676 & 9.052 & 5.875 & 6.885 & 3.038 & 4.097 & 0.308 \\
$y$ & 0.211 & 2.725 & 1.933 & 3.242 & 7.858 & 6.061 & 7.173 & 3.082 & 0.894 & 1.203 \\
$e$ & 0.575 & -0.647 & -1.346 & -1.566 & 1.194 & -0.186 & -0.288 & -0.044 & 3.203 & -0.895
\end{tabular}



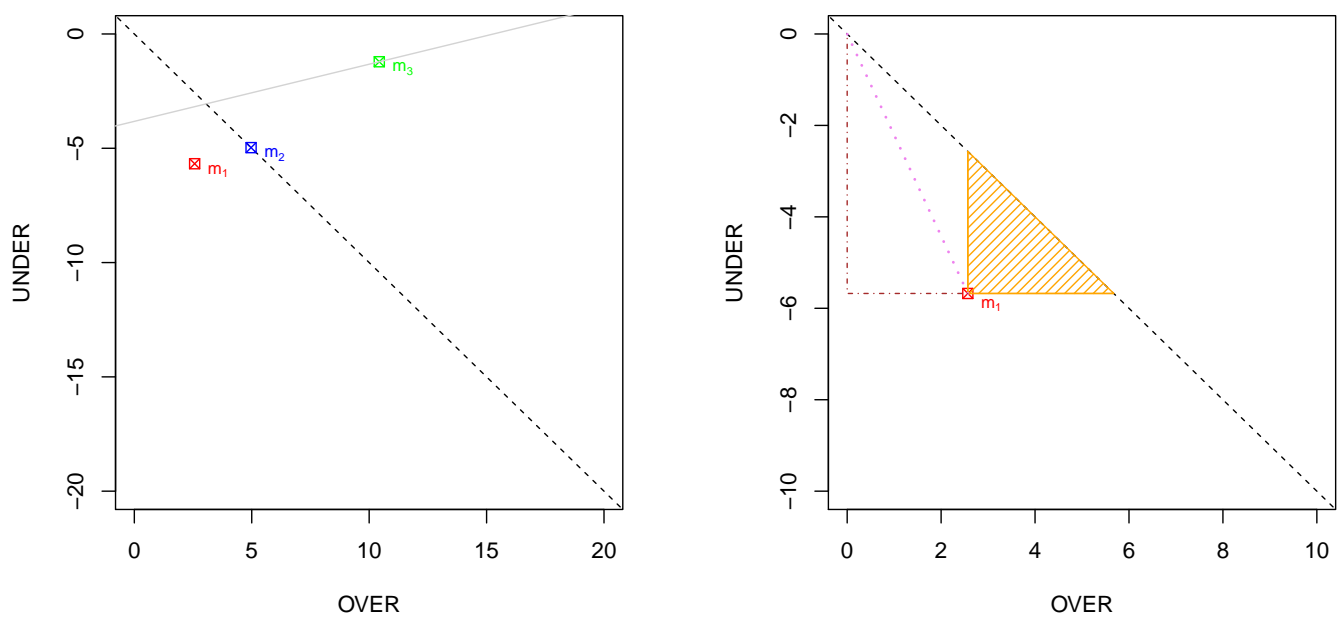

Figure 1: Left: RROC space and the representation for regression models $m_{1}$ (in red), $m_{2}$ (in blue) and $m_{3}$ (in green) in examples 1, 2 and 3. The diagonal (dashed black) shows where UNDER and $O V E R$ are equal. Model $m_{2}$ has zero error bias $(\mu(\mathbf{e})=0)$. We also show the first isometric line (solid light grey) corresponding to $\alpha=0.8$ (slope $=0.25$ ) touching any of the three models. Right: RROC space with regression model $m_{1}$ (in red) in example 1 (note that the RROC space has been zoomed in now for both $x$-axis and $y$-axis). We show several metrics that can be plotted on the RROC space: the sum of the length of the two dot-dashed segments in brown is exactly the absolute error $(A E)$ : 8.25 ; the length of the dotted violet segment equals the unweighted macroaverage squared error $(u S E)$ : 6.23 ; the length of either the horizontal or vertical orange segment connecting the point $m_{1}$ with the diagonal is exactly the total error bias $(E B=\mu(e) \cdot n=O V E R+$ $U N D E R):-3.107$; and the area of the orange-shaded triangle is clearly half of the squared error bias $\left(\frac{1}{2}(\mu(e) \cdot n)^{2}=\frac{1}{2}(O V E R+U N D E R)^{2}\right): 4.827$.

The sum of over-estimations (OVER) is 4.972 while the sum of under-estimations (UNDER) is -4.972 . This regression model finds an equilibrium between over and under-estimations (it is unbiased, since $\mu(\mathbf{e})=0$ ). However, the MAE (0.9944) and the MSE (1.7619) are worse than for $m_{1}$ in example 1.

Model $m_{2}$ is also shown in Figure 1 (left). Clearly it is on the diagonal.

Finally let us consider a third model:

Example 3. Consider a regression model $m_{3}$ as follows:

\begin{tabular}{c|cccccccccc} 
& 1 & 2 & 3 & 4 & 5 & 6 & 7 & 8 & 9 & 10 \\
$\hat{y}$ & 1.253 & 4.232 & 1.734 & 5.325 & 6.842 & 9.325 & 8.232 & 3.525 & 1.352 & 1.778 \\
$y$ & 0.211 & 2.725 & 1.933 & 3.242 & 7.858 & 6.061 & 7.173 & 3.082 & 0.894 & 1.203 \\
$e$ & 1.042 & 1.507 & -0.199 & 2.083 & -1.016 & 3.264 & 1.059 & 0.443 & 0.458 & 0.575
\end{tabular}

In this case, the sum of over-estimations (OVER) is 10.431 while the sum 
of under-estimations (UNDER) is -1.215. This regression model clearly overestimates (it has a positive error bias, since $\mu(\mathbf{e})=0.9216>0$ ). The MAE (1.165) and the MSE (2.12) show that this model is worse than models $m_{1}$ and $m_{2}$.

From each point in RROC space, we can derive several metrics very easily, as seen in Figure 1 (right) for model $m_{1}$. We have that $M A E=0.825=$ $(O V E R-U N D E R) / n$, so $A E$ is just half the perimeter of the rectangle that each point creates with the RROC heaven $(0,0)$. In other words, the $A E$ is just the Manhattan distance to RROC heaven. The diagonal of this rectangle (the Euclidean distance) is just given by $\left.\sqrt{(} O V E R^{2}+U N D E R^{2}\right)$. This measure, which we call $U S E$ (as an unweighted macro-averaged version of the total squared error, $S E$ ), is interesting in itself, because highly penalises models with a high imbalance between over and under-estimations, and can be seen as a measure of 'symmetric calibration'. Finally, the total error bias $E B=\mu(e) \cdot n$ equals the length of the two parallel (horizontal and vertical) segments from the model point to the diagonal.

In RROC space we denote the regression model always outputting $\infty$ and the model always outputting $-\infty$ as the (trivial) extreme regression models, which fall at $(\infty, 0)$ and $(0,-\infty)$ respectively in RROC space.

\subsection{RROC space isometrics}

We have mentioned above that (half) the perimeter of the rectangle from RROC heaven to the regression model corresponds to the absolute error. Can we extend this observation to the asymmetric loss? The following straightforward lemma shows that total asymmetric absolute loss can be calculated graphically as the sum of the distance to the $y$-axis $(O V E R=0)$ and to the $x$-axis $(U N D E R=0)$, using the appropriate asymmetry factor $\alpha$.

Lemma 1. The total asymmetric absolute loss is given by:

$$
L=\sum_{i} \ell_{\alpha}^{A}\left(\hat{y}_{i}, y_{i}\right)=2(1-\alpha) \cdot O V E R-2 \alpha \cdot U N D E R
$$

Proof.

$$
\begin{aligned}
L=\sum_{i} \ell_{\alpha}^{A}\left(\hat{y}_{i}, y_{i}\right) & =\sum_{i}\left\{2 \alpha\left(y_{i}-\hat{y}_{i}\right) \text { if } \hat{y}_{i}<y_{i}, 2(1-\alpha)\left(\hat{y}_{i}-y_{i}\right) \text { otherwise }\right\} \\
& =\sum_{i}\left\{2 \alpha\left(-e_{i}\right) \mid e_{i}<0\right\}+\sum_{i}\left\{2(1-\alpha)\left(e_{i}\right) \mid e_{i}>0\right\} \\
& =-2 \alpha \cdot \text { UNDER }+2(1-\alpha) \cdot \text { OVER }
\end{aligned}
$$


Clearly, for $\alpha=0.5$, we have that this is the absolute error. This also shows that the closer we are to RROC heaven $(0,0)$ (in terms of a Manhattan distance) the better. All this leads to loss isometrics:

Definition 5. RROC isometrics are defined by varying $t$ over:

$$
2(1-\alpha) \cdot \text { OVER }-2 \alpha \cdot U N D E R=t
$$

The following proposition just gets the slope of each parallel isometric:

Proposition 2. Given an isometric $2(1-\alpha) \cdot$ OVER $-2 \alpha \cdot U N D E R=t$, the slope only depends on $\alpha$ and is given by:

$$
\text { slope }=\frac{1-\alpha}{\alpha}
$$

Proof. By isolating the variable $U N D E R$ we have:

$$
\begin{aligned}
\text { UNDER } & =\frac{-2(1-\alpha) \cdot \text { OVER }+t}{-2 \alpha}=\frac{1-\alpha}{\alpha} \cdot \text { OVER }+\frac{-2 t}{\alpha} \\
& =\text { slope } \cdot \text { OVER }+ \text { intercept }
\end{aligned}
$$

The slope is then given by the first term $\frac{1-\alpha}{\alpha}$

Clearly, for $\alpha=0$ (under-estimations have no cost) we have infinite slope. For $\alpha=1$ (over-estimations have no cost), we have a slope 0 . This notion of isometric is very similar to the notion already present in ROC analysis for classification [18]. In fact, this means that we can slide isometrics (moving $t$ ) to find optimal points in RROC space, in the very same way as we do in ROC space.

Let us illustrate this. Figure 1 (left) shows the RROC space and the regression models $m_{1}, m_{2}$ and $m_{3}$ in examples 1,2 and 3 respectively. We also consider the operating condition $\alpha=0.8$, meaning that under-estimations are 4 times more expensive than over-estimations. This $\alpha$ leads to a slope of 0.25 . By sliding through all the parallel isometric lines from the one crossing the RROC heaven $(0,0)$ to the first isometric touching a point corresponding to any model, we touch at $(10.431,-1.215)$ first. In fact, the intercept is given by isolating it from the line equation (eq. 1 above), which, in this case, leads to -3.82275 . The line $U N D E R=$ 0.25 . OVER -3.82275 is then shown on Figure 1 (left), touching regression model $m_{3}$. Even though model $m_{3}$ has a worse mean (symmetric) absolute error than $m_{1}$ (and $m_{2}$ ), for this operating condition $\alpha$ it leads to lower total asymmetric 

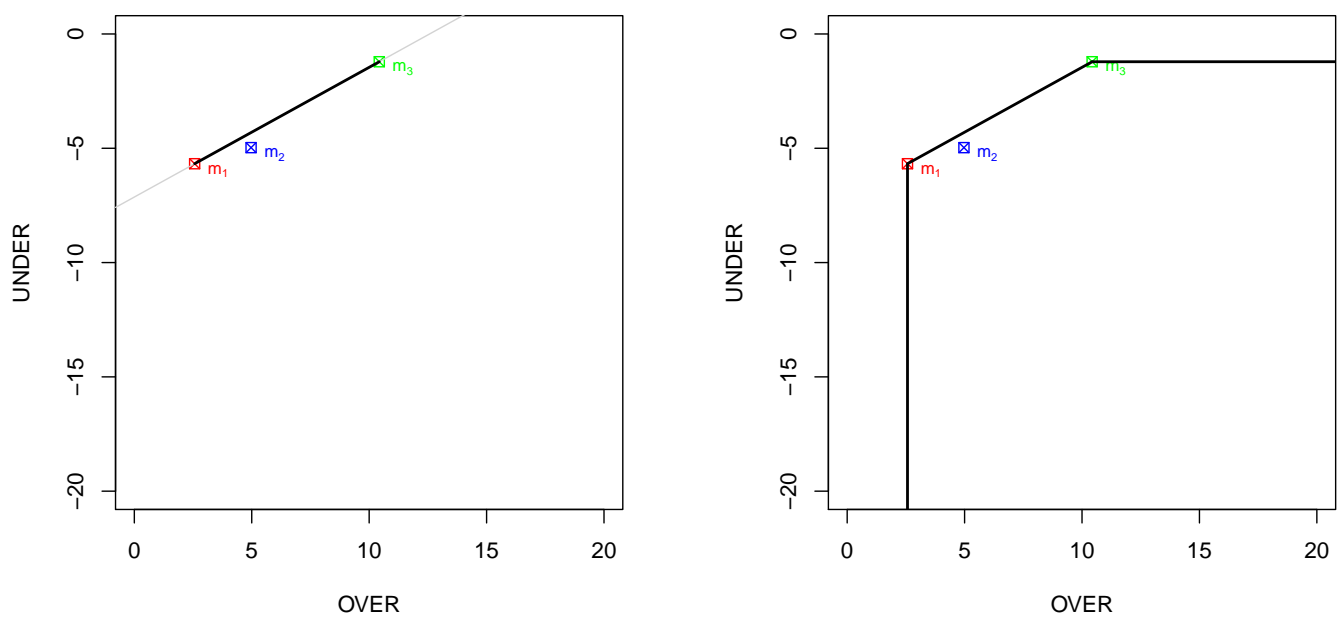

Figure 2: The three models as in Figure 1 (left). Left: by considering any model which can be constructed by randomly choosing (with any bias) between models $m_{1}$ and $m_{3}$, we can show a segment of models (in solid black). Right: completing this for any other two models -including the extreme models at $(0,-\infty)$ and $(\infty, 0)$ - we can derive the convex hull (shown in solid black).

absolute error. Namely, while $m_{1}$ has a loss of $2(1-\alpha) \cdot$ OVER $-2 \alpha \cdot U N D E R=$ $0.4 \cdot 2.569-1.6 \cdot(-5.676)=10.1092$, we have that $m_{3}$ has a loss of $2(1-\alpha)$. OVER $-2 \alpha \cdot U N D E R=0.4 \cdot 10.431-1.6 \cdot(-1.215)=6.1164$.

\subsection{Hybrid models, dominance and convex hull}

Another construction that is also originally present in ROC analysis for classification is the notion of hybrid models. Given any two models, we can construct a hybrid model by randomly choosing each prediction from any of both models using a (possibly biased) coin. We can also do this in regression. Figure 2 (left) shows the isometric (in light grey) passing through models $m_{1}$ and $m_{3}$. The solid black segment connecting both models shows that any model along the segment can be constructed. More precisely, each point in that segment would represent the expected value of a model constructed by choosing between the models in a random (biased) way. Consequently, we can just connect both points since any point in between is technically achievable (at least in expectation).

For instance, a hybrid model using a random (in this case unbiased) choice between these two models leads to points $O V E R=(2.569+10.431) / 2=6.5$ and $U N D E R=(-5.676-1.215) / 2=-3.45$ on expectation, which is exactly on the middle of the segment. This point represents that we use $m_{1}$ half of the times and 
$m_{2}$ the other half ${ }^{1}$. This is like ROC analysis for classification.

In this particular case, we just draw a line between the point representing $m_{1}:(2.569,-5.676)$ and the point representing $m_{3}:(10.431,-1.215)$, leading to $U N D E R=0.567 \cdot$ OVER -7.134 . From this slope of 0.567 , we just calculate $\alpha=\frac{1}{1+\text { slope }}=0.638$. Obviously, for this $\alpha$ both models have the same loss. $L\left(m_{1}\right)=(1-0.638) \cdot 2.569+0.638 \cdot 5.676=4.551$ and $L\left(m_{3}\right)=(1-0.638)$. $10.431+0.638 \cdot 1.215=4.551$.

Given these two models, we say that, for slopes lower than 0.567 (asymmetries $\alpha$ greater than 0.638 ), model $m_{3}$ dominates, while we have that model $m_{1}$ dominates for the rest of operating conditions.

This leads to the notion of dominance and convex hull. In fact, after connecting all the points by the segments representing the hybrid models, and also including the extreme classifiers at $(0,-\infty)$ and $(\infty, 0)$, we can calculate the convex hull. Any model under the convex hull can be discarded, in the same way as traditional ROC analysis does. Figure 2 (right) shows the convex hull of the three models and the extreme models. We see that model $m_{2}$ can be discarded. It cannot be optimal for any operating condition.

\section{RROC curves}

In ROC analysis for classification, we can tweak the predictions of a crisp classifier by changing the predicted class of a random percentage of examples. With this, we can move the classifier in ROC space. However, this just moves the classifier along the two straight lines that connect the original point with the points at $(0,0)$ and $(1,1)$ (the trivial, or extreme, classifiers).

In general, however, in ROC analysis, curves are constructed by the use of soft classifiers, i.e., classifiers which output a rank, score or probability estimation. By moving a threshold from the lowest possible value to the highest possible value (or vice versa) we get many possible crisp classifiers, each of them represented by a point in ROC space, leading to a ROC curve.

Interestingly, in RROC space, we do not need soft regression models in order to create a curve. It is just sufficient to use a shift, which works as a parallel concept to the notion of threshold. For each example we can get a modified prediction

\footnotetext{
${ }^{1}$ Remarkably, this is very different to averaging both models. For instance, if we calculate the (unweighted) average of $m_{1}$ and $m_{3}$ we have an error vector $\mathbf{e}=$ $\langle 0.375,1.053,0.094,-0.040,-0.491,1.093,-0.497,0.402,0.824,0.242\rangle$, with $O V E R=4.081$ and $U N D E R=-1.0265$. This point is not on the segment.
} 
as $\hat{y}^{\prime} \leftarrow \hat{y}+s$, where $s$ is the shift. Although there are, as we will see, many ways of determining this shift, it seems natural to consider first that $s$ is constant, i.e., that we apply the same value for all the examples.

Definition 6. Given a regression model $m$, a (constant-)shifted regression model, denoted by $m\langle s\rangle$, is the result of adding the same shift $s$ to all its predictions, i.e., $\hat{y}^{\prime} \leftarrow \hat{y}+s$ for all predictions $\hat{y}$.

The use of a shift $s$ can lead to models with different values of UNDER and $O V E R$, denoted by $U N D E R\langle s\rangle$ and $O V E R\langle s\rangle$, and lower (or higher) loss. In fact, given the asymmetric absolute loss with an operating condition $\alpha$, and assuming a (constant-)shifted regression model, the optimal shift choice method $s^{*}(\alpha)$ is derived from lemma 1 as:

$s^{*}(\alpha)=\underset{s}{\arg \min } \sum_{i} \ell_{\alpha}^{A}\left(\hat{y}_{i}, y_{i}\right)=\underset{s}{\arg \min }\{2(1-\alpha) \cdot$ OVER $\langle s\rangle-2 \alpha \cdot U N D E R\langle s\rangle\}$

This means that the original bias of the model is irrelevant as we can use $s^{*}(\alpha)$ to eliminate the bias (for symmetric losses) or to minimise an asymmetric loss. Actually, this shift can be moved from the lowest possible value $(-\infty)$ to the maximum possible value $(\infty)$. All this leads to the notion of RROC curve.

Definition 7. Given a regression model $m$, its RROC curve using a (constant) shift $i$ given by plotting all the models $m\langle s\rangle$ with $s$ ranging in $[-\infty, \infty]$.

In fact, the function $s^{*}(\alpha)$ is non-decreasing, and we only need to explore the shifts from $s^{*}(0)=\arg \min _{s} 2 \cdot$ OVER $\langle s\rangle=-\max (\mathbf{e})$ to $s^{*}(1)=\arg \min _{s} 2$. $U N D E R\langle s\rangle=-\min (\mathbf{e})$. For example 1 , these values are $s^{0}(0)=-0.598$ and $s^{1}(0)=2.162$. We can instantly plot the curves point-wise, by just using a sufficient number of values for $s$ in this range. However, there is a more direct way of plotting and analysing the RROC curve. This is what we do next.

\subsection{Algorithm for drawing RROC curves}

We can realise that if we move the shift from $s_{1}$ to $s_{2}$ and no example changes from $O V E R$ to $U N D E R$ or vice versa, then the increment/decrement in $O V E R$ and $U N D E R$ is linear, as the following proposition shows:

Proposition 3. Given a model $m$, for any two shifts $s_{1}$ and $s_{2}$ such that the examples for which $m\left\langle s_{1}\right\rangle$ and $m\left\langle s_{2}\right\rangle$ over-estimate are the same (and hence the rest that under-estimate are also the same for both), then for any other shift $s_{3}$ with $s_{1} \leq s_{3} \leq s_{2}$ we have that the points (OVER, UNDER) for the three models $m\left\langle s_{1}\right\rangle$, $m\left\langle s_{2}\right\rangle$ and $m\left\langle s_{3}\right\rangle$ lie on the same straight line. 
Proof. We have that OVER for $m\left\langle s_{1}\right\rangle$ is calculated as: OVER $\left\langle s_{1}\right\rangle=\sum_{i}\left\{e_{i}+\right.$ $\left.s_{1} \mid e_{i}+s_{1}>0\right\}$ while OVER for $m\left\langle s_{2}\right\rangle$ is calculated as: OVER $\left\langle s_{2}\right\rangle=\sum_{i}\left\{e_{i}+\right.$ $\left.s_{2} \mid e_{i}+s_{2}>0\right\}$. Since, by assumption, the examples which over-estimate are the same for $m\left\langle s_{1}\right\rangle$ and $m\left\langle s_{2}\right\rangle$, let us call this number $n_{o}$. The previous two expressions can then be rewritten as:

$$
\begin{aligned}
& \text { OVER }\left\langle s_{1}\right\rangle=n_{o} s_{1}+\sum_{i}\left\{e_{i} \mid e_{i}+s_{1}>0\right\} \\
& \text { OVER }\left\langle s_{2}\right\rangle=n_{o} s_{2}+\sum_{i}\left\{e_{i} \mid e_{i}+s_{1}>0\right\}
\end{aligned}
$$

Note that the second term is also rewritten with $s_{1}$, since the elements are the same. Also, since the examples which over-estimate are the same for $s_{1}$ and $s_{2}$ they are necessarily the same for every $s_{3}$ with $s_{1} \leq s_{3} \leq s_{2}$. So, we have:

$$
O V E R\left\langle s_{3}\right\rangle \triangleq n_{o} s_{3}+\sum_{i}\left\{e_{i} \mid e_{i}+s_{1}>0\right\}
$$

We can see that these three co-ordinates only differ on the first term, which is linearly related to $s\left(s_{1}, s_{2}\right.$ or $\left.s_{3}\right)$. We can obtain similar expressions for $U N D E R\left\langle s_{1}\right\rangle$, $U N D E R\left\langle s_{2}\right\rangle$ and $U N D E R\left\langle s_{3}\right\rangle$ and their $n_{u}$ examples. The three points are related by a linear term on $s$, expressed as $\left(n_{o} s, n_{u} s\right)$, so they lie on the same line.

From proposition 3 we can introduce a very simple algorithm to draw RROC curves (Algorithm 1).

Figure 3 (left) shows a RROC curve using this algorithm for model $m_{1}$ in example 1. The points where the slope of the RROC Curve change are called vertex points, and the rest of points fall onto the segments. Consequently a RROC Curve for a regression model applied to a dataset with $n$ instances has $n+2$ vertex points (typically, only $n$ are visible on the plot, because two are the extreme points) and $n+1$ segments, denoted by $\overline{i, i+1}$ with $i=1 \ldots n+1$.

In case there are some ties in the error vector, some vertex points and segments collapse into a single point. Let us see this:

Example 4. Consider a regression model $m_{4}$ as follows:

\begin{tabular}{c|cccccccccc} 
& 1 & 2 & 3 & 4 & 5 & 6 & 7 & 8 & 9 & 10 \\
$\hat{y}$ & 0.123 & 1.221 & 1.845 & 4.573 & 8.558 & 7.392 & 5.669 & 1.578 & 0.806 & 1.245 \\
$y$ & 0.211 & 2.725 & 1.933 & 3.242 & 7.858 & 6.061 & 7.173 & 3.082 & 0.894 & 1.203 \\
$e$ & -0.088 & -1.504 & -0.088 & 1.331 & 0.700 & 1.331 & -1.504 & -1.504 & -0.088 & 0.042
\end{tabular}




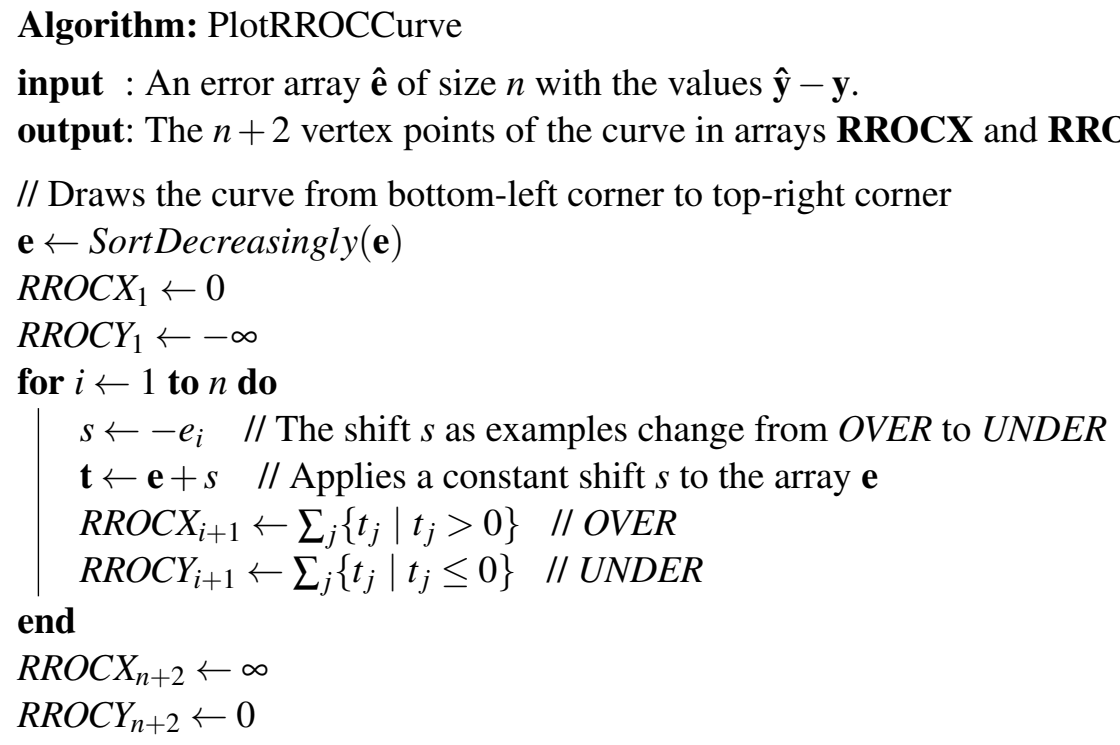

Algorithm 1: Draws a RROC curve. We use boldface for arrays notation.

We see a triple tie between examples 1, 3 and 9, another triple tie between examples 2, 7 and 8, and a double tie between examples 4 and 6 .

Figure 3 (right) only has five points as there are only 5 different error values.

\subsection{Properties: slope and convexity}

From the new RROC curve, we may want to calculate the slopes of each segment, in order to exactly determine where each possible isometric (and asymmetry $\alpha$ ) would lead to on the curve. This can be done very easily:

Lemma 4. The slope of each segment $\overline{i, i+1}$ in the RROC curve is given by $(n+$ $1-i) /(i-1)$, with $i=1 \ldots n+1$.

Proof. Let us assume there are no ties in the error vector. As shown in proposition 3 , there is one example changing from $U N D E R$ to $O V E R$ (from bottom-left to topright) at each vertex point. At the first vertex point $i=1$, all the examples are under-estimated, and the shift change moves along an infinite slope. For the next vertex point $i=2$, we have $n-1$ under-estimated examples and 1 over-estimated example. This means that any shift increment corresponds to one unit right and $n-1$ units up. This is a slope of $n-1$. By induction, this leads to $(n+1-i) /(i-$ $1)$, with the last segment having 0 slope. If there are ties, then more than one example will change from under-estimation to over-estimation at a time. 

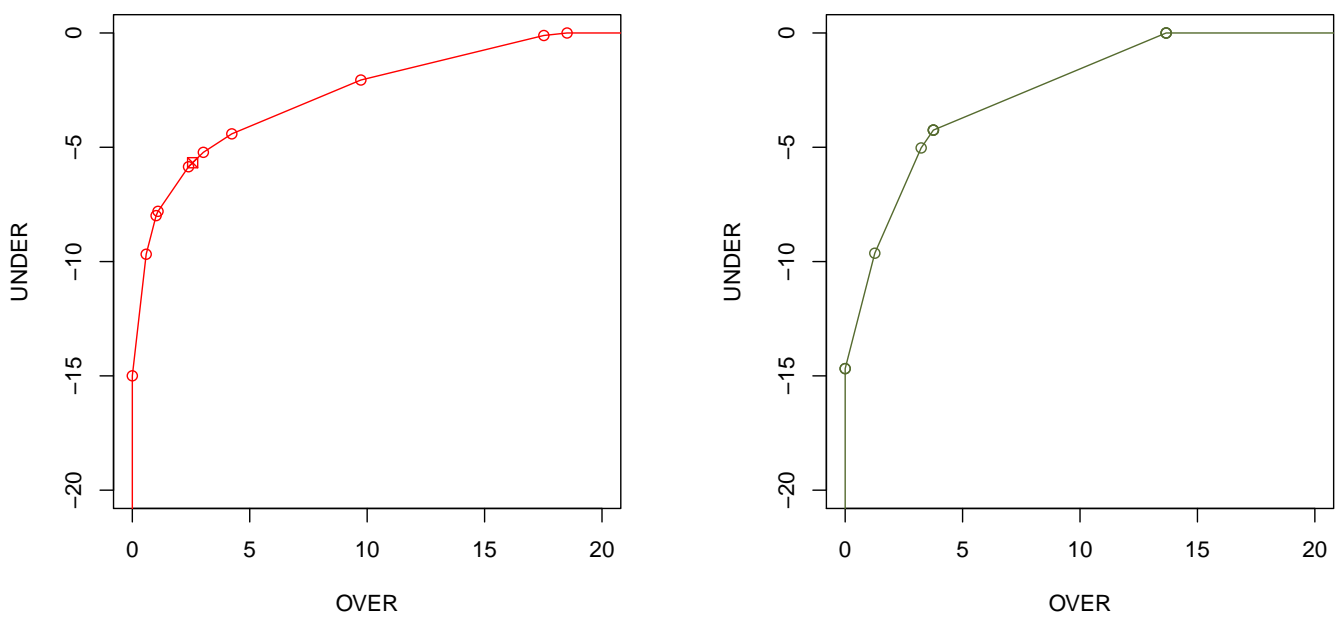

Figure 3: Left: Model $m_{1}$ in example 1 drawn as a RROC curve by changing the shift. Vertex points (10 in this case, since the two extremes are not visible in the plot) are shown as small circles. The curve is then composed of 11 segments. The original shift $(s=0)$ is still represented with a small square and lies on a segment between two vertex points. Right: Model $m_{4}$ in example 4 drawn as a RROC curve by changing the shift. The model has several errors with the same value (two triple ties and a double tie), so the number of distinct visible points is reduced from $n=10$ to $n-2-2-1=5$.

Thus, and somewhat surprisingly, given a dataset with a fixed number of examples, all regression models (without ties) will have exactly the same slopes. The difference between the curves will be given by the length of the segments, not their slopes. From the equation $\frac{1-\alpha}{\alpha}=$ slope in proposition 2 relating asymmetries and slopes, we have that each segment $\overline{i, i+1}$ corresponds to $\alpha=\frac{1}{\text { slope+1 }}$, leading to $\alpha=\frac{i-1}{n}$ with $i=1 \ldots n+1$.

Finally, we can see immediately that RROC curves are convex:

Proposition 5. For every regression model, the RROC Curve is convex.

Proof. It is direct from lemma 4 since the sequence of the segment slopes of the curve $(n+1-i) /(i-1)$ is non-increasing.

This property is very useful when working with curves (e.g., for calculating their dominance regions) and it also allows the direct calculation of the optimal shift as for eq. 2. The convexity of a single RROC curve does not mean that the notion of convex hull seen in the previous section is useless. More on the contrary, whenever we have more than one model, we can see concavities. Figure 4 (left) precisely shows this, and the convex hull is shown in Figure 4 (right). 

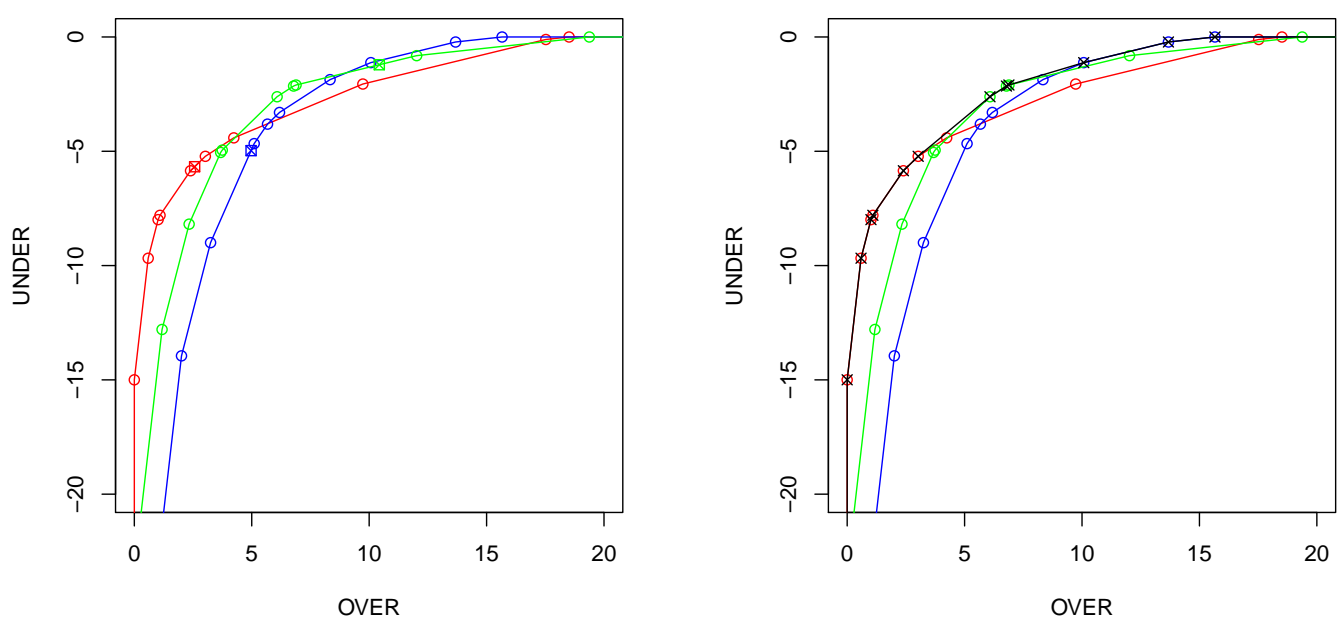

Figure 4: The left plot shows the three models $m_{1}$ (red), $m_{2}$ (blue) and $m_{3}$ (green) in examples 1, 2 and 3 drawn as RROC curves by changing the shift. Note that in this case model $m_{3}$ cannot be rejected, because there are regions where it is optimal. If we select the best portions from the three models we see concavities, which can be resolved by the use of a convex hull (shown on the right) in black. There are 12 visible points (represented as black crosses) on the convex hull: 6 from $m_{1}$ (red), 3 from $m_{3}$ (green), and 3 from $m_{2}$ (blue).

\subsection{Related and alternative graphical tools}

Before further analysing RROC curves, it is appropriate to take a look at other curves and graphical tools that are used for the analysis of regression models. Regression Error Characteristic (REC) curves [3] are, by far, the most successful attempt to bring ROC analysis to regression. The idea is based on plotting the percentage of examples (accuracy) whose predictions are inside a tolerance level $\varepsilon$. As the tolerance level increases on the $x$-axis the accuracy increases on the $y$-axis, so REC curves are always non-decreasing. Alternatively, REC curves can be seen as a plot of the cumulative distribution function of the prediction error. Similarly to ROC curves, several models can be represented in the same space, so that dominance regions can be determined. The crucial point about REC curves is what the operating condition means. In the case of REC curves, the operating condition is the maximum tolerance level that is admitted for predictions. This is useful in applications where the maximum admitted error can vary from time to time. If a context is very strict on errors we can use the models that perform better in that range. On the other hand, if we are more flexible on errors, we can choose a different model. We will plot some REC curves in section 6.

Regression Error Characteristic Surfaces (RECS) [51] are an extension of REC curves where the cumulative distribution of the dependent variable is set as an 
additional dimension [51]. In this way, REC surfaces (if the loss function is the absolute deviation) show the joint cumulative distribution function of the errors and the target variable. The operating condition is then a pair of tolerance level $\varepsilon$ and the value of the dependent variable. This is useful if we have different tolerance levels depending on the output variable. In fact, it is easy to make a section of the REC surface to account for relative performance, as, e.g., admitting an error of at most $25 \%$.

Despite their usefulness, none of the previous approaches focusses on the problem of asymmetric loss functions and the notion of operating condition as a parameter of the loss function ${ }^{2}$. As a result, we do not have the dual (TPR vs. FPR) view of ROC analysis (where both axes have the same dimension and commensurable meaning), from where the notion of loss isometrics can be derived. Also, we think that the problem of asymmetry in regression is arguably more common than the problem of limited tolerance. Nonetheless, we see RROC curves and REC curves as complementary tools, which either (or both) can be used depending on the kind of operating conditions we want to deal with.

There are other more classical plots for analysing regression models, such as scatter plots (predicted vs. actual), residual density plots and residual boxplots [33]. While these plots make it possible to see model asymmetries, it is very difficult to compare several models and determine the dominance regions. Also, Figure 5 (left) will also show a residual density plot. In section 6, we will show scatter plots and residual density plots and compare them to RROC curves and REC curves.

Finally, as there is a point-to-line (or point-to-point [29]) correspondence between ROC curves and cost curves [12] in classification, we can also consider the definition of cost curves for regression. The cost space for regression (RCOST) is defined as a plot where the expected loss (e.g., the asymmetric absolute loss) is shown on the $y$-axis for a range of operating conditions (e.g., the asymmetry $\alpha$ ) on the $x$-axis. We will also show a RCOST curve in section 6 .

\section{Areas and metrics}

RROC curves, as in ROC analysis, can be especially useful for analysing models under different operating conditions and select the best one for a single operating condition or a region an interval of operating conditions. Also, we can create

\footnotetext{
${ }^{2}$ In the so-called utility-based regression [41], the asymmetric absolute loss function (Lin-Lin) is discussed, but no specific plot is introduced to deal with this asymmetry.
} 
hybrids through the notion of convex hull. Nonetheless, in ROC analysis we are also interested in evaluating models that can work well for a wide range of operating conditions. One measure that gives us a good indication of a classifier performing well in a wide range of operating conditions is the Area Under the ROC Curve $(A U C)$. Can we develop a similar measure for RROC curves? The following definition introduces such a measure:

Definition 8. The Area Over the RROC Curve (AOC) is defined as follows:

$$
A O C \triangleq-\int_{0}^{\infty} U N D E R \text { dOVER }=\int_{-\infty}^{0} \text { OVER dUNDER }
$$

Lower values for $A O C$ are better. This area can be calculated very easily by using the sum of the $n+1$ upward trapeziums given between the elements 1 and $\mathrm{n}+2$ from $R R O C X$ and $R R O C X$ in algorithm 1. Actually, for models always outputting finite values, this can be calculated from 2 to $n$, since the extreme trapezium 1 to 2 has area 0 and the trapezium $n+1$ to $n+2$ as well, so we only need to sum $n-1$ trapeziums. Consequently:

$$
A O C=\sum_{i=2}^{n}-\frac{R R O C Y_{i+1}+R R O C Y_{i}}{2}\left(\operatorname{RROCX}_{i+1}-\operatorname{RROCX}_{i}\right)
$$

As the RROC space is unbounded, only the area over the curve makes sense:

Proposition 6. For any regression model $m$ which always outputs finite values, the $A O C$ is finite.

Proof. Since the model $m$ always outputs finite values, there is a shift $s_{o}$, such that for any shift $s \leq s_{O}$ we have that $O V E R=0$ and there is also a shift $s_{u}$, such that for any shift $s \geq s_{u}$ we have that $U N D E R=0$. This means that the curve touches (and stays at) both the $x$-axis and the $y$-axis. Then the area is finite.

For the three models $m_{1}, m_{2}$ and $m_{3}$ in Figure 4 (left), the $A O C$ is 56.1387, 88.0933 and 63.9295, respectively. What is the meaning of these figures? To answer this question, let us first consider the 'best' possible model in terms of $A O C$ (a perfect square with top-left corner at the RROC heaven $(0,0)$ ). This model means that there is a shift that achieves 0 error. This is rarely the case, except for datasets with one single example (where there is always a shift getting 0 loss). It is also very rare to have a dataset for which all the examples have the same error, another possible situation where we would have $A O C=0$. 
We could also think about the relation of $A O C$ to other metrics, For instance, a model with very high $M S E$ or $M A E$ could, in principle, have $A O C=0$. This would suggest that the shift was very badly chosen. This phenomenon mirrors what happens with classical ROC analysis; we can have bad accuracy for a model with optimal $A U C$ by choosing a bad threshold.

A possible way to look at $A O C$ is as the expected value of the total underestimation given a distribution for the total over-estimation. Alternative, if we look at the distance to RROC heaven, we can see $A O C$ as an aggregate of the macroaverage squared error $(n \cdot u S E)$ with a distribution which depends on the model, which is similar to one recent interpretation given to AUC [24]. Other interpretations as an aggregation of expected loss may be possible ${ }^{3}$, as it has happened with $A U C$ recently, where new interpretations have been introduced [20, 28].

Having said all this, the idea of the $A O C$ being related to the distribution of errors seems more appealing. If we have a compact error distribution, then $A O C$ will be low because many shifts will be able to place most errors close to 0 . If we have a sparse error distribution, then $A O C$ will be high. One classical measure of dispersion is precisely the variance, defined and decomposed as follows:

Definition 9. The error variance $\sigma(\mathbf{e})^{2}\left(\right.$ or, simply, $\left.\sigma^{2}\right)$ is defined as:

$$
\sigma(\mathbf{e})^{2} \triangleq \frac{\sum_{i=1}^{n}\left(e_{i}-\mu(\mathbf{e})\right)^{2}}{n}=\frac{\sum_{i=1}^{n} e_{i}^{2}}{n}-\mu(\mathbf{e})^{2}=\mu\left(\mathbf{e}^{2}\right)-\mu(\mathbf{e})^{2}=M S E-\mu(\mathbf{e})^{2}
$$

where $\mu(\mathbf{e})$ (or, simply, $\mu$ ) represents the mean of the vector $\mathbf{e}$.

Note that we define the population error variance, by dividing by $n$ (instead of $n-1)$. The last term in definition 9 is just the classical $M S E$ decomposition as the sum of the squared mean error bias $\left(\mu^{2}\right)$ and the error variance $\left(\sigma^{2}\right)$.

Quite surprisingly, the observation that the $A O C$ and the error variance are related can be made extremely precise, as the following theorem shows:

Theorem 7. The area over the RROC curve equals the population variance $\sigma^{2}$ of the errors multiplied by a factor $n^{2} / 2$ which is independent of the model. Namely:

$$
A O C=\frac{n^{2}}{2} \sigma^{2}
$$

\footnotetext{
${ }^{3}$ We suggest some possible pathways for exploration. Since $A O C$ is related to the magnitude of predictions (and errors), it cannot be directly related to rank-based correlation measures in regression. However, it could be related to this sum $\sum_{y_{1}, y_{2}}\left(\hat{y}_{1}-\hat{y}_{2} \mid y_{1}>y_{2}\right)$, which would work as a counterpart of the Wilcoxon-Mann-Whitney interpretation of the $A U C$.
} 
The proof is found in the appendix.

Corollary 8. If the model is unbiased (i.e. $\mu(\mathbf{e})=0$ ) then:

$$
A O C=\frac{n^{2}}{2} M S E
$$

The proof of this corollary is direct from theorem 7 and definition 9 (error variance decomposition). These important results provide a natural interpretation of the $A O C$ and its connection with error variance and MSE. Also, AOC (and indirectly $M S E$ ) can be interpreted as a measure of performance over a range of asymmetry parameters $\alpha$, provided we choose the shift optimally (eq. 2).

For the models $m_{1}, m_{2}$ and $m_{3}$ in examples 1,2 and 3 we have a variance of $1.1228,1.7619$ and 1.2786 respectively. The $A O C$ is $56.1387,88.0933$ and 63.9295 respectively. Since $m_{2}$ is unbiased, its $M S E$ is precisely 1.7619 , its error variance. The constant factor is $n^{2} / 2=50$ in the three cases.

In general, we can show the $M S E$ decomposition (the squared error bias and the error variance) in RROC space. In Figure 1 (right) we saw that the area of the shadowed triangle is exactly half the squared error bias. By summing this area to the $A O C$ we have half the squared error $(S E)$. This again draws a parallelism with classification, where a perfectly calibrated model has 0 calibration loss, and $M S E$ is just given by the refinement loss, recently connected with $A U C$ [28]. In regression, we have just seen that a model with no bias leads to a $M S E$ which is just the error variance, now connected with $A O C$.

Given this link between the area over the RROC curve and the error variance, we can also explore the link with an error density plot. As we can see in Figure 5 (left), there is a high correspondence between the density plots and the RROC curve, but the cumulative character of the RROC curve makes the latter smoother.

Note that this connection between $A O C$ and the error variance indicates that it is the dispersion that counts when adapting our models to cost-sensitive situations with asymmetries, and not the position, which can be ignored if the optimal shift is chosen for each particular operating condition. This again shows a parallel with ROC analysis, where the absolute values of the scores do not affect the $A U C$. Only their order matters. Here, for RROC curves, the position of the mean error (the error bias) does not affect the $A O C$, only the dispersion of the error.

Interestingly, the $n^{2}$ factor in theorem 7 also suggests that a scaled representation of RROC curves could be done by dividing both $x$-axis and $y$-axis by $n$, i.e., plotting $O V E R / n$ against $U N D E R / n$. This would make the curves independent of the number of examples and could be the standard representation in many appli- 

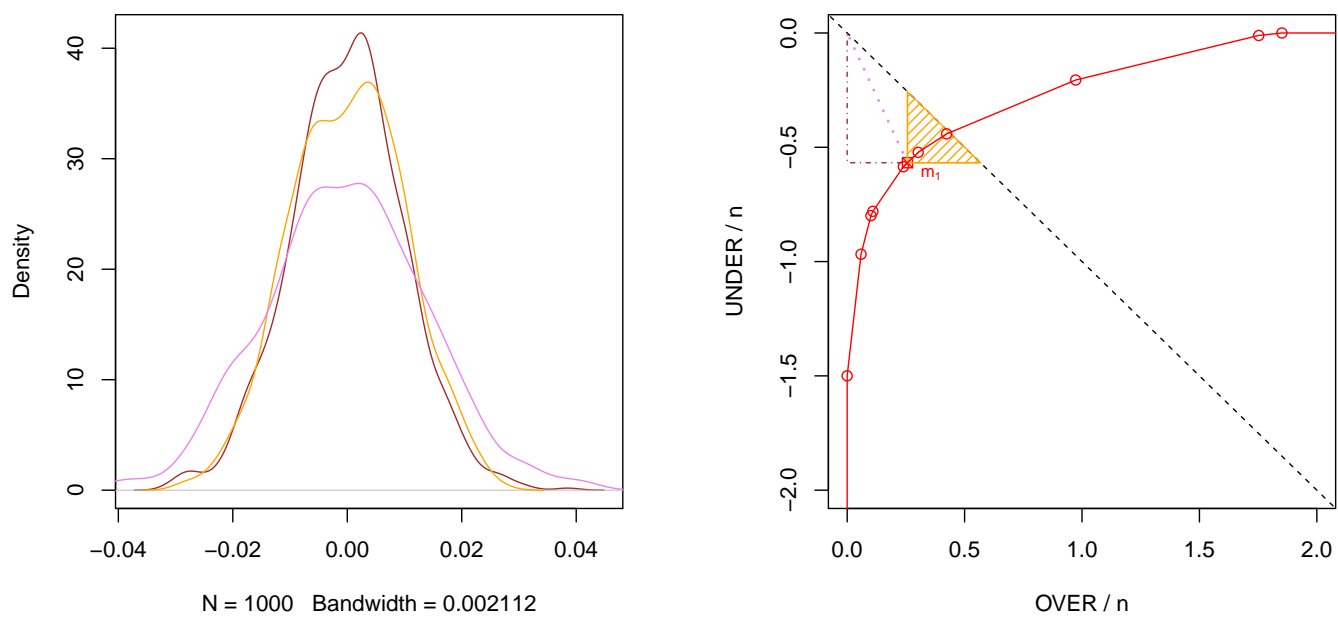

Figure 5: Left: The error density plots of the same three models as in Figure 4. We can see that similar error density functions will produce similar RROC curves. Here, more peaked density functions mean better RROC curves. Right: Model $m_{1}$ as in example 1 drawn as a normalised RROC curve, using a normalised scale for the $x$-axis and $y$-axis ( $\mathrm{n}=10$ examples). Compare with Figure 3 (left). Now we can see the average metrics of those shown in Figure 1 (right).

cations, especially when the number of examples in the datasets may vary or we may even compare several models (or the same model) against different datasets (with different sizes). Figure 5 (right) shows the same plot as Figure 3 (left) but normalising by the number of examples (in this case $n=10$ ). It also shows the same metrics as in Figure 1 (right), but now the magnitudes correspond to the mean versions (MAE, MSE, $u M S E$ and $M E B$ ).

Finally, the derivation of an area from a curve is not exclusive of ROC curves (or RROC curves). Some of the curves discussed in section 4.3 have associated areas with particular meanings. For instance, the AOC for REC curves have been shown [3] to be a lower estimation of $M A E$ if the error function is the absolute error and a lower estimation of $M S E$ if the error function is the squared error. As a result, we can say that if the error bias is zero, then the AOC of a REC curve is always lower than or equal to the AOC of a RROC curve. Nonetheless, it is important to note that the AOC is the sample error variance (more precisely a linear function of it), and not an estimation. Also, these two areas for REC and RROC curves represent different things and may serve different purposes. In order to see this, we include the AOC for the REC curves and the RROC curves in the real example that follows. 


\section{A case study}

Cost asymmetries and biased predictions are an issue in most application areas where regression is used: sales forecasting, inventory control, position and speed tracking, pose estimation, survival analysis, consumption estimation, demand prediction, weather forecasting, series analysis and econometrics, among many others. Project effort estimation is one application area where over-estimations and under-estimations may have different costs depending on the situation. As a real example, we will work with a software engineering project effort dataset. In this area in particular [45], "underestimating software projects can have detrimental effects on business reputation, competitiveness, and performance" while "overestimated projects can result in poor resource allocation and missed opportunities to take on other projects" [5]. This phenomenon is extensible to other areas as well $[53,2,6,49,39]$. The relation between under-estimation and over-estimation costs can be tuned by the project manager depending on the context.

We will use a dataset comprising 145 projects from the Computer Science Corporation (CSC). This dataset has been thoroughly discussed and analysed in [33]. We use the (compact) version of the dataset published in [33], with attributes "client code" ( $c c)$, "project type" ( $p t)$ and "adjusted function points" ( $a f p$ ) as predictive variables, and the "actual effort" (ae) in hours as the dependent variable 4 .

Typically, the evaluation metrics used for this and other effort estimation problems are [33]: (1) the mean magnitude relative error (MMRE), which is a normalisation of the absolute error, (2) "the proportion of project estimates within $25 \%$ of the actual", denoted as "Pred(25)", and (3) boxplots for the residuals. The first one is a special case of the asymmetric absolute loss used in RROC curves (assuming $\alpha=0.5$ ), while the third one is used to "show whether or not the estimates are biased [...] and whether the model has a tendency to under- or over-estimate" [33]. With RROC curves we will be able to see both things (and more, such as dominance, isometrics and areas) with the same plot. The second one seems to be closely related to the concept of tolerance in REC curves, although REC curves use a notion of absolute tolerance, while Pred(25) refers to a notion of relative

\footnotetext{
${ }^{4}$ This attribute setting is similar to "model 1" in [33]. We investigated the logarithmic transformation of the numerical variables afp and ae (as done in some models in [33]), but we did not find any significant improvement for any of the regression methods we used. For the two categorical variables we merged all the values (including missing values) with less than 10 instances into a single merger label (so that new values cannot appear on the test set if they have not previously appeared on the training set). Finally, we removed the outlier example 102, following [33].
} 

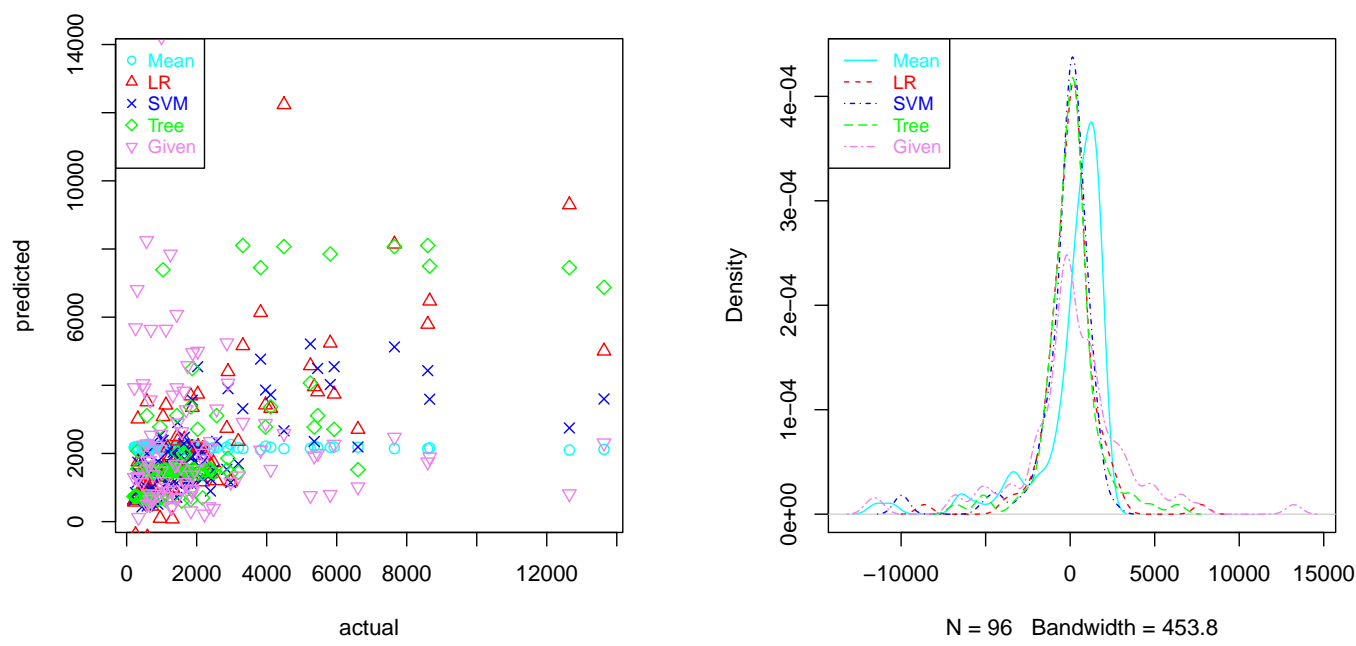

Figure 6: Five regression models on the work dataset. Left: a scatter plot. Right: density plot. We see that $L R$ and $S V M$ are more peaked than the rest (and they peak at a residual of 0), with almost no tails, while the others seem to perform worse, especially Given.

tolerance. As a result, RROC curves are very suitable for this kind of problem in conjunction with other approaches, such as REC curves.

We considered the following experimental scenario. We randomly split the dataset into a work dataset (2/3 of the data) for model training and selection and a deployment dataset ( $1 / 3$ of the data) for final evaluation. We built several models with the work dataset using 10-fold cross-validation. As a custom procedure (see, e.g., [3]), the aggregated results of the 10 trials were used to build the plots (RROC and REC curves), estimate the metrics and dominance regions. Then, the 10 trials were discarded and the whole work dataset was used to retrain the models, as usual in cross-validation. Finally, the models were evaluated for the deployment dataset under several operating conditions (the asymmetry cost parameter $\alpha$ ).

We compared five methods: Mean is the mean of the dependent variable for the training set, $L R$ is a linear regression using the function $\mathrm{lm}$ in $\mathrm{R}$ [40], SVM is a support vector machine using the function $\mathrm{ksvm}$ in the $R$ package kernlab, and Tree is a regression tree using the function rpart in the $R$ package rpart. In addition, for comparison, we also included a method known as Given, which is provided by [33] as an estimation method used by CSC ("first estimate").

Figure 6 shows the results of these five methods on the work dataset (the test values for the 10 trials) using two common plots: a scatter plot and a residual density plot. While these two plots show some relevant information, it is very difficult to compare several models with them, see the tolerance levels or the asymmetries. 

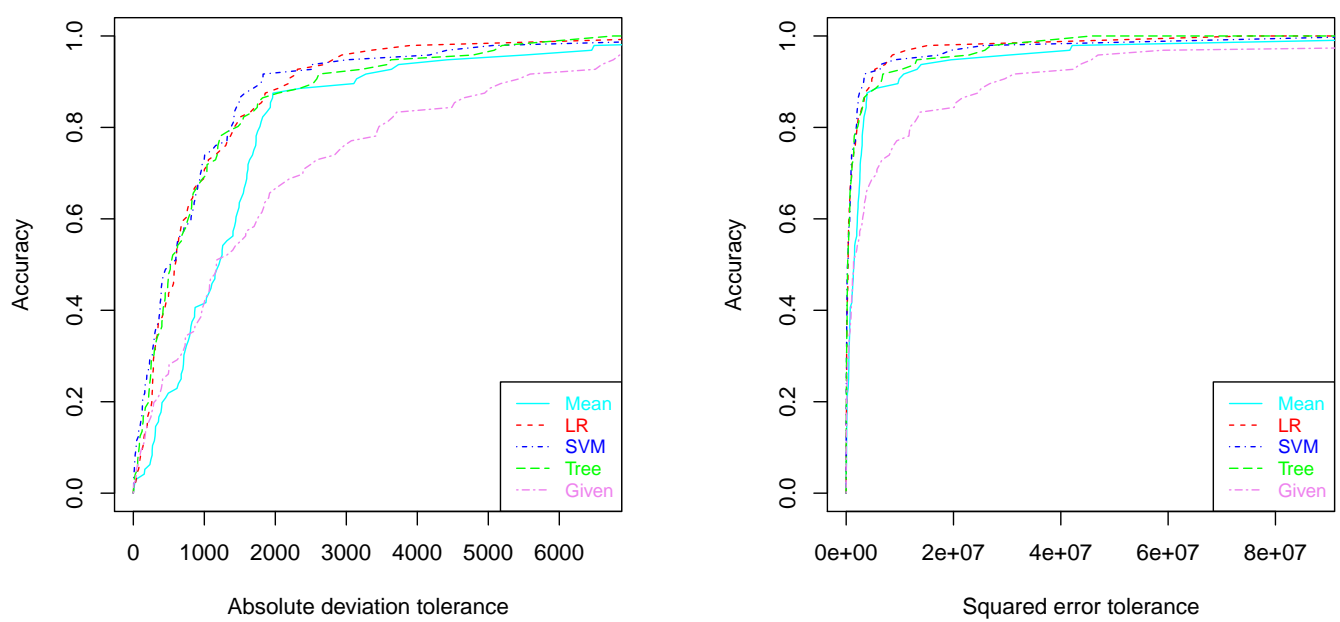

Figure 7: REC Curves. Left: using absolute error (absolute deviation). Right: using squared error.

Things improve significantly when we plot the REC curves (described in section 4.3) for the five models (see Figure 7). We see that the Mean model and especially the Given model behave much worse for almost all ranges of error tolerance. Also, e.g., we can see on Figure 7 (left) that if we set our absolute deviation tolerance at 2000 hours, then the best model seems to be $S V M$. However, REC curves are not devised to show asymmetry information and we cannot see the bias either.

Figure 8 shows the corresponding RROC curve. Here we can clearly see the asymmetries. For low over-estimations (low values of $\alpha$ ), Mean and SVM are better, while $L M$ is better for low under-estimations (high values of $\alpha$ ). Note that the curves implicitly assume the best shift for each operating condition $\alpha$. By using eq. $2, s^{*}(\alpha)$ can be exactly calculated on a sample (in this case the work dataset). The ultimate goodness of this mapping on a deployment dataset will be given by the quality of the RROC curve estimation (we will explore this below).

We can also compare some metrics that can be derived from the plots we have seen so far (see Table 1). Interestingly, we can also see the contribution of each of these measures graphically and whether the differences between two models are originated uniformly from a wide range of operating conditions (as happens with the difference in $M A E$ between $L R$ and Tree) or it conceals a cancellation for two different dominance regions (as happens with the difference in $M A E$ between $L R$ and $S V M$ ). This can only be seen on the RROC curves, and not by any global metric (including $A O C$ ).

Once the analysis has been done on a work dataset, it is time to see how our findings and selections behave on a deployment dataset. From work to deploy- 

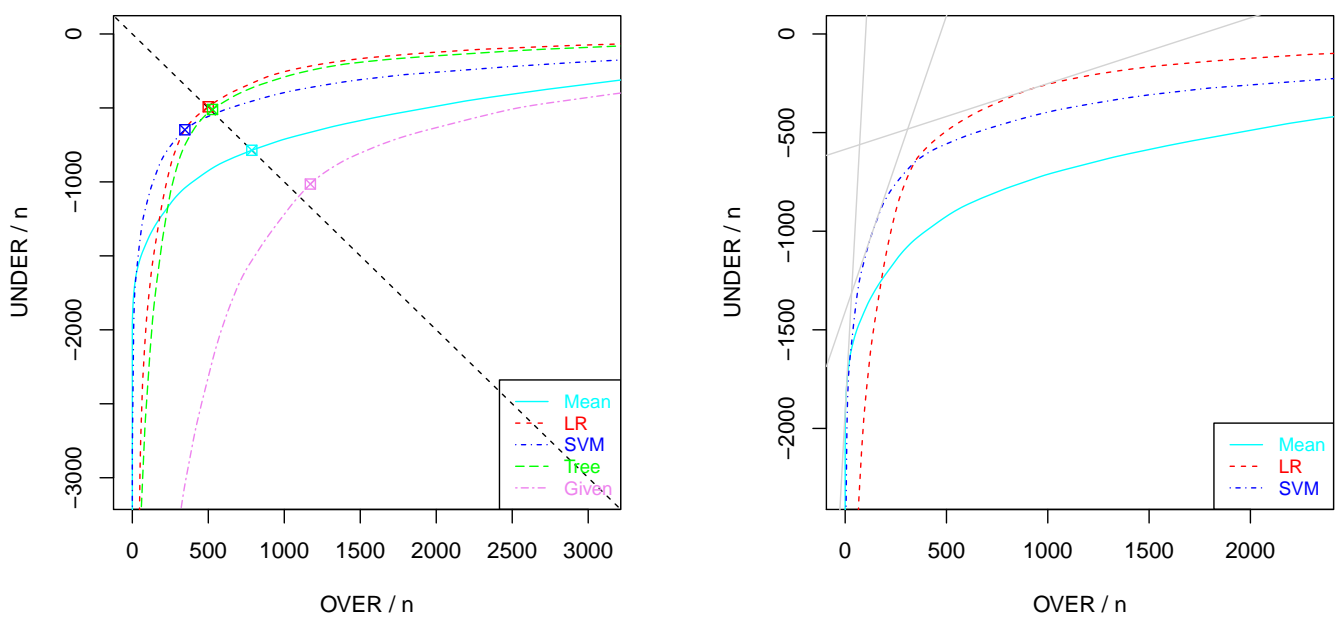

Figure 8: RROC Curves for the work dataset (using the 10 folds of a cross-validation process). Left: the RROC curve for the five models, showing a negative bias for $S V M$ and a positive bias for Given, while the other three models are unbiased (almost on the diagonal). Right: the same (zoomed-in) RROC curve where the Tree and Given models have been removed, since they can be safely discarded (as far as the curves are well estimated). We show three isometrics corresponding to $\alpha=0.05,0.25,0.75$, in grey, clockwise from bottom left to top right. By taking the slopes where the curves cross, we can easily calculate the dominance intervals: $\alpha \in[0,0.103]$ for the Mean model, $\alpha \in[0.103,0.453]$ for the $S V M$ model, and $\alpha \in[0.453,1]$ for the $L R$ model.

ment, we need to keep three things: (1) the five models, (2) the dominance regions (see Figure 8 ) and (3) the five shift mappings $s^{*}(\alpha)$ corresponding to each model (that can be stored into an array for a range of $\alpha \in[0,1])^{5}$. Note that elements 2 and 3 are available if we just keep the segments of the RROC curves. With all these elements, the procedure at deployment time is simple: (1) recognise the operating condition $\alpha$, (2) look at the dominance regions and see which model $m$ is best for that $\alpha$, (3) take the mapping $s^{*}$ for $m$ to calculate the shift $s^{*}(\alpha)$, and (4) apply the shift to $m$ to make the predictions.

The effectiveness of the above procedure will depend on the accurate estimation of the RROC curve. For instance, the dominance regions for the work dataset could differ from those in the deployment dataset. We can see whether this happens in this example by comparing the curves shown on figures 8 and 9 and the intervals identified in their captions. Actually, the regisions are similar. But the ultimate quality criterion must be whether this procedure can choose the model that

\footnotetext{
${ }^{5}$ There are more elaborate methods, such as those of [1][55], using non-constant shifts, or even using soft regressors [25], which can also work with other loss functions.
} 


\begin{tabular}{r|rrrrrr} 
& $M A E$ & $M S E$ & $M E B$ & $A O C^{R E C_{A E}}$ & $A O C^{R E C_{S E}}$ & $2 A O C^{R R O C} / n^{2}=\sigma^{2}$ \\
Mean & 1572.6 & 5803921 & 0.1 & 1496.6 & 4865235 & 5803921 \\
LR & 991.7 & 2714267 & 7.3 & 931.4 & 2169130 & 2714214 \\
SVM & 993.4 & 3533795 & -301.8 & 916.7 & 2646860 & 3442698 \\
Tree & 1033.1 & 2866122 & 17.5 & 999.0 & 2586698 & 2865817 \\
Given & 2186.2 & 11348130 & 157.1 & 2122.0 & 10558810 & 11323462
\end{tabular}

Table 1: Averaged 10-fold cross-validation results on the work dataset. We see several metrics for the five models of our case study: MAE, MSE, MEB, the area over the REC curve using absolute error, the area over the REC curve using squared error and the normalised version of the area over the RROC curve (which equals the error variance). While the AOC of the REC curves are underestimations of the MAE and MSE metrics (as shown by [3]), we see that the AOC of the RROC curve is just the $M S E$ minus the squared $M E B$ as given by the decomposition in eq. 9 .

(using $s^{*}$ ) minimises the loss function for each possible condition. This is nicely reproduced by the RCOST curve (introduced at the end of section 4) shown on the right of Figure 9. This shows that the procedure has worked almost perfectly, by choosing the best model for almost all over the range.

On other occasions we may be interested in part of the range of $\alpha \in[0,1]$. For instance, in this software effort problem we may be told that operating conditions usually range between $\alpha \in[0.33,0.8]$, i.e., from cases where an over-estimation is up to 2 times (0.33 vs. 0.67$)$ more costly than an under-estimation to cases where under-estimation is up to 4 times $(0.2$ vs. 0.8$)$ more costly than an overestimation). Then we only need to look at this range of operating conditions on Figure 8, and see that it is enough to keep the $L R$ model and the SVM model.

Figure 9 also shows a close (and very interesting) relation between RROC curves and RCOST curves. While RCOST curves are obviously better for locating the expected loss for each operating condition, RROC curves have more information: under-estimation and over-estimation. In fact, from them, we can get the loss, but not the other way round. Also, the $A O C$ corresponds to the variance of the residual, while the area under the RCOST curve is only meaningful as an expected loss for a uniform distribution of operating conditions. Another advantage of RROC space over RCOST space is that by varying the isometrics (from straight lines to any other function) we could see the notion of dominance for several asymmetric loss functions, while cost curves would need to be redrawn. This comparison is extremely similar to that in classification [12] [29] and unveils a potential for further exploration.

Finally, Table 2 shows the quality metrics for the deployment dataset. The comparison with Table 1 shows that there are important differences in global metrics. Despite this, the model selection that we have performed with RROC analysis has still been quite robust. 

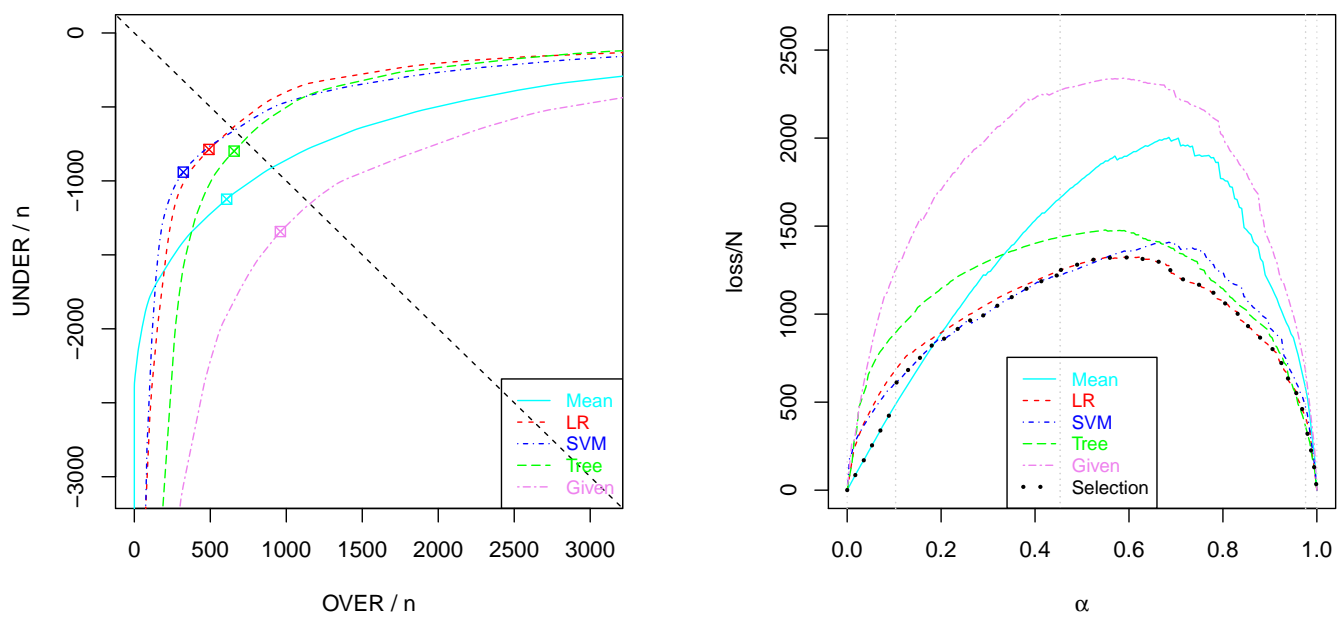

Figure 9: Left: the RROC curve for the five models (previously trained with the whole work dataset) on the deployment dataset. The dominance intervals are now: $\alpha \in[0,0.159]$ for the Mean model, $\alpha \in[0.159,0.548]$ for the $S V M$ model, and $\alpha \in[0.548,0.938]$ for the $L R$ model (there is a small interval for the Tree model for $\alpha \in[0.938,1])$. These are approximate to those found for the work dataset (see Figure 8). Right: a RCOST curve shows the actual asymmetric loss for $\alpha \in[0,1]$. The dotted curve in black shows the results obtained by the selection of models performed for the work dataset, using the dominance regions in Figure 8 (shown as grey vertical lines) and the map function $s^{*}$ obtained from the work dataset. We see that piecewise composition of the choices made by this procedure has led to a very good choice for this deployment dataset.

\section{Discussion}

We said in the introduction that there is no such a thing as the 'canonical' ROC space for regression, corresponding exactly to the ROC space for classification, since regression and classification are very different tasks. Having said this, we think that the RROC space, curves and analysis that we have introduced in this paper present so many parallelisms and share so many common notions and procedures, that their curves could reasonably be called the ROC curves for regression, with arguably more support than other previous attempts. We have seen that the notions of operating condition, cost asymmetry, RROC space, points, segments, RROC heaven, RROC isometrics, hybrid models, convexity, dominance, convex hull, curves, shift choice methods, etc., derive smoothly and work almost the same as in the classification case, so the practitioners which are used to ROC curves can directly apply their expertise on ROC analysis to regression quite easily.

There are also some caveats. First, while we have argued that asymmetric costs are common in many regression applications, there might also be some problems where asymmetries are not important. Second, when asymmetry is important the 


\begin{tabular}{r|rrrrrr} 
& $M A E$ & $M S E$ & $M E B$ & $A O C^{R E C_{A E}}$ & $A O C^{R E C_{S E}}$ & $2 A O C^{R R O C} / n^{2}=\sigma^{2}$ \\
Mean & 1731.4 & 7305474 & -516.2 & 1642.5 & 6646875 & 7039046 \\
LR & 1278.2 & 4200835 & -296.2 & 1197.2 & 3659776 & 4113120 \\
SVM & 1263.1 & 5134660 & -618.4 & 1174.8 & 4503110 & 4752242 \\
Tree & 1455.5 & 5119506 & -141.8 & 1376.3 & 4532698 & 5099411 \\
Given & 2303.8 & 12689846 & -381.3 & 2203.8 & 11748335 & 12544431
\end{tabular}

Table 2: Similar to Figure 1 but calculated on the deployment dataset.

operating condition may be unknown on deployment time ${ }^{6}$. Third, keeping several models and choosing the best one depending on the operating condition seems a reasonable way to adapt predictions to the new context, but there is always the alternative approach of retraining the model (provided that we can use specialised regression techniques for each cost function [11, 39]). This is known as the reframing/retraining dilemma. Fourth, on occasions the data at hand may be insufficient to do a good estimation of the RROC curves (even if we use cross-validation or confidence bands). Hence the rejection of regression models and the identification of dominance regions may be statistically unreliable. These four caveats are not new to RROC curves, but inherited from the very nature of ROC analysis as a method of choosing the best model given the operating condition.

We now point out other more specific limitations of RROC curves and suggest some possible solutions, which can be used as start points for future work. The first issue that could be explored and generalised is the very notion of operating condition. We have only considered the loss asymmetry while, in classification, the class distribution can also be integrated (along with the cost proportion) in what is usually referred to as skew. In regression, the distribution of the output value (and not only the loss asymmetry) may also be considered part of the operating condition as well. This integration does not seem to be direct as a single parameter, but it is worth being investigated as a $3 \mathrm{D}$ plot, possibly in the same way REC surfaces extend REC curves by including the cumulative distribution of the dependent variable as an additional dimension [51]. Also, the combination of REC curves and RROC curves could be explored.

A (related) second issue is the use of other loss functions. For instance, instead of an asymmetric absolute error, we could use an asymmetric squared error QuadQuad. This would lead to non-straight isometrics in the RROC curve, but the

\footnotetext{
${ }^{6}$ Related to these two first caveats, we note that there has been some work to explore whether costs should or should not be asymmetric and even determine their parameters from the dataset (e.g., [9], for the Lin-Lin cost function, as the one used here).
} 
basic ideas would remain. Again, plotting different isometrics in RROC space for many different loss functions (Lin-Lin, Quad-Quad, Lin-Exp, Quad-Exp, etc.) would closely resemble the celebrated paper [18] on isometrics for ROC curves in classification.

The most common application of RROC curves is the local selection of models according to an operating condition, as we have seen in a real scenario in section 6 , instead of the selection of models by the use of global metrics. Nonetheless, a third important avenue of future work would be to further investigate the connection with the error variance we have unveiled here and to analyse the relation of $A O C$ with other global metrics. We think that RROC curves represent the expected loss for a range of operating conditions on one side, and the distribution of the error on the other side (as the $A O C$ has been shown to be equivalent to the error variance). The derivation of global or partial (see, e.g., the use of partial $A U C$ in [42]) areas using some knowledge about the ranges and/or distributions of the operating conditions may be useful in many applications, where we do not know the exact operating condition, but we may still have some information about some asymmetries being more likely than others. In general, there may be important connections to be unveiled between regression techniques trying to minimise the error variance (instead of squared error) and those classification techniques trying to maximise the AUC (instead of accuracy) [15]. Also, the link between $A O C$ and the $M S E$ decomposition could be related to the recently discovered equivalence of $A U C$ to the refinement loss term of the MSE decomposition using the ROC curve [28]. So we anticipate a plethora of connections between RROC curves and many other performance metrics in regression, as has been done for classification in the past years [16, 24, 20, 27, 28].

Finally, the relation between classification and regression could be better understood, since any scoring classifier (producing probabilities, likelihoods or other kinds of scores) can also be examined in terms of asymmetry as if they were regression models. In fact, some datasets (e.g., in signal detection) are presented with a quantitative output, modelled with regression and ultimately converted into categorical decisions (alarm, failure, etc.) with a (varying) threshold.

Overall, we think that RROC curves could become a fundamental tool in the assessment, improvement and deployment of regression models. In order to facilitate their use in real applications, we have developed a library for plotting RROC curves $^{7}$, the intercepts and dominance regions, calculating their areas and deriv-

\footnotetext{
${ }^{7}$ The software, in R [40], is at http://users.dsic.upv.es/ㄱ 7ejorallo/RROC/.
} 
ing their convex hulls. The library also includes tools for plotting REC curves and RCOST curves, as well as the code for reproducing all the examples in this paper. The availability of software, the ubiquitous appearance of asymmetric losses in regression applications, and the success of ROC analysis for classification in the past decades suggests that RROC curves may soon become mainstream in all the areas where ROC analysis has shown to be useful: medicine, bioinformatics, statistics, machine learning and pattern recognition.

\section{Acknowledgments}

I would like to thank Peter Flach and Nicolas Lachiche for some very useful comments and corrections on earlier versions of this paper, especially the suggestion of drawing normalised curves (dividing $x$-axis and $y$-axis by $n$ ). This work was supported by the MEC/MINECO projects CONSOLIDER-INGENIO CSD2007-00022 and TIN 201021062-C02-02, GVA project Prometeo/2008/051, the COST - European Cooperation in the field of Scientific and Technical Research IC0801 AT, and the REFRAME project granted by the European Coordinated Research on Long-term Challenges in Information and Communication Sciences \& Technologies ERA-Net (CHIST-ERA), and funded by the respective national research councils and ministries.

\section{References}

[1] G. Bansal, A. Sinha, and H. Zhao. Tuning data mining methods for cost-sensitive regression: A study in loan charge-off forecasting. J. Management Information System, 25:315-336, December 2008.

[2] A. P. Basu and N. Ebrahimi. Bayesian approach to life testing and reliability estimation using asymmetric loss function. Journal of statistical planning and inference, 29(1-2):21-31, 1992.

[3] J. Bi and K. P. Bennett. Regression error characteristic curves. In Twentieth International Conference on Machine Learning (ICML-2003). Washington, DC, 2003.

[4] A. P. Bradley. The use of the area under the ROC curve in the evaluation of machine learning algorithms. Pattern Recognition, 30(7):1145 - 1159, 1997.

[5] L. C. Briand and I. Wieczorek. Resource estimation in software engineering. Encyclopedia of software engineering, 2002.

[6] M. Cain and C. Janssen. Real estate price prediction under asymmetric loss. Annals of the Institute of Statistical Mathematics, 47(3):401-414, 1995.

[7] P. F. Christoffersen and F. X. Diebold. Further results on forecasting and model selection under asymmetric loss. J. of applied econometrics, 11(5):561-571, 1996.

[8] P. F. Christoffersen and F. X. Diebold. Optimal prediction under asymmetric loss. Econometric Theory, 13:808-817, 1997.

[9] M. A. Clatworthy, D. A. Peel, and P. F. Pope. Are analysts' loss functions asymmetric? Journal of Forecasting, 31(8):736-756, 2012. 
[10] S. Crone. Training artificial neural networks for time series prediction using asymmetric cost functions. In 9th Intl. Conf. on Neural Information Processing, 2002.

[11] M. Demetrescu. An extension of the gauss-newton algorithm for estimation under asymmetric loss. Computational statistics \& data analysis, 50(2):379-401, 2006.

[12] C. Drummond and R.C. Holte. Cost Curves: An Improved Method for Visualizing Classifier Performance. Machine Learning, 65:95-130, 2006.

[13] C. Elkan. The foundations of Cost-Sensitive learning. In Bernhard Nebel, editor, Proceedings of the seventeenth International Conference on Artificial Intelligence (IJCAI-01), pages 973-978, San Francisco, CA, 2001.

[14] T. Fawcett. An introduction to ROC analysis. Pattern Recognition Letters, 27(8):861-874, 2006.

[15] C. Ferri, P. Flach, and J. Hernández-Orallo. Learning decision trees using the area under the ROC curve. In International Conference on Machine Learning, pages 139-146, 2002.

[16] C. Ferri, J. Hernández-Orallo, and R. Modroiu. An experimental comparison of performance measures for classification. Pattern Recognition Let., 30(1):27-38, 2009.

[17] C. Ferri, J. Hernández-Orallo, and M. Salido. Volume under the ROC surface for multi-class problems. Machine Learning: ECML 2003, pages 108-120, 2003.

[18] P. Flach. The geometry of ROC space: Understanding machine learning metrics through ROC isometrics. In Machine Learning, Proceedings of the Twentieth International Conference (ICML 2003), pages 194-201, 2003.

[19] P. Flach, H. Blockeel, C. Ferri, J. Hernández-Orallo, and J. Struyf. Decision support for data mining. Data Mining and Decision Support, pages 81-90, 2003.

[20] P. Flach, J. Hernández-Orallo, and C. Ferri. A coherent interpretation of AUC as a measure of aggregated classification performance. In Proceedings of the 28th International Conference on Machine Learning, ICML2011, 2011.

[21] J. E. Goin. ROC curve estimation and hypothesis testing: applications to breast cancer detection. Pattern Recognition, 15(3):263 - 269, 1982.

[22] C. W. J. Granger. Prediction with a generalized cost of error function. Operational Research Quarter, 20:199-207, 1969.

[23] C. W. J. Granger. Outline of forecast theory using generalized cost functions. Spanish Economic Review, 1(2):161-173, 1999.

[24] D. J. Hand. Measuring classifier performance: a coherent alternative to the area under the ROC curve. Machine learning, 77(1):103-123, 2009.

[25] J. Hernández-Orallo. Probabilistic reframing for context-sensitive regression. submitted, preliminary version at http://arxiv.org/abs/1211.1043, 2012.

[26] J. Hernández-Orallo, C. Ferri, N. Lachiche, and P. Flach. The 1st workshop on ROC analysis in artificial intelligence (ROCAI-2004). ACM SIGKDD Explorations Newsletter, 6(2):159-161, 2004.

[27] J. Hernández-Orallo, P. Flach, and C. Ferri. Brier curves: a new cost-based visualisation of classifier performance. In Proceedings of the 28th International Conference on Machine Learning, ICML2011, 2011.

[28] J. Hernández-Orallo, P. Flach, and C. Ferri. A unified view of performance metrics: Translating threshold choice into expected classification loss. Journal of Machine 
Learning Research (JMLR), 13:2813-2869, 2012.

[29] J. Hernández-Orallo, P. Flach, and C. Ferri. ROC curves in cost space. Machine Learning, 2013.

[30] W. Khreich, E. Granger, A. Miri, and R. Sabourin. Iterative boolean combination of classifiers in the ROC space: An application to anomaly detection with HMMs. Pattern Recognition, 43(8):2732 - 2752, 2010.

[31] W. Khreich, E. Granger, A. Miri, and R. Sabourin. Adaptive ROC-based ensembles of HMMs applied to anomaly detection. Pattern Recognition, 45(1):208 - 230, 2012.

[32] Y. Kim, K. A. Toh, A. Beng J. Teoh, H. L. Eng, and W. Y. Yau. An online AUC formulation for binary classification. Pattern Recognition, 45(6):2266 - 2279, 2012.

[33] B. Kitchenham, Shari Lawrence P., B. McColl, and S. Eagan. An empirical study of maintenance and development estimation accuracy. Journal of systems and software, 64(1):57-77, 2002.

[34] W. J. Krzanowski. ROC curves for continuous data, volume 111. Chapman \& Hall/CRC, 2009.

[35] T. A. Lasko, J. G. Bhagwat, K. H. Zou, L. Ohno-Machado, et al. The use of receiver operating characteristic curves in biomedical informatics. Journal of biomedical informatics, 38(5):404-415, 2005.

[36] L. B. Lusted. Signal detectability and medical decision-making. Science, 171:12171219, 1971.

[37] H. Mamitsuka. Selecting features in microarray classification using ROC curves. Pattern Recognition, 39(12):2393 - 2404, 2006.

[38] C. Marrocco, R. P. W. Duin, and F. Tortorella. Maximizing the area under the ROC curve by pairwise feature combination. Pattern Recognition, 41(6):1961-74, 2008.

[39] A. J. Patton and A. Timmermann. Testing forecast optimality under unknown loss. Journal of the American Statistical Association, 102(480), 2007.

[40] R Team et al. R: A language and environment for statistical computing. R Foundation for Statistical Computing, Vienna, Austria, 2012.

[41] R. Ribeiro. Utility-based regression. PhD thesis, Dep. computer Science, Faculty of Sciences - University of Porto, 2011.

[42] M. T. Ricamato and F. Tortorella. Partial AUC maximization in a linear combination of dichotomizers. Pattern Recognition, 44(10-11):2669 - 2677, 2011.

[43] S. Rosset, C. Perlich, and B. Zadrozny. Ranking-based evaluation of regression models. Knowledge and Information Systems, 12(3):331-353, 2007.

[44] C. M. Schubert, S. N. Thorsen, and M. E. Oxley. The ROC manifold for classification systems. Pattern Recognition, 44(2):350 - 362, 2011.

[45] D. G. Silva, M. Jino, and B. T. de Abreu. Machine learning methods and asymmetric cost function to estimate execution effort of software testing. In Software Testing, Verification and Validation (ICST), pages 275-284. IEEE, 2010.

[46] A. Srinivasan. Note on the location of optimal classifiers in n-dimensional ROC space. Technical Report PRG-TR-2-99, Oxford University Computing Laboratory, Wolfson Building, Parks Road, Oxford., 1999.

[47] J. A. Swets. Measuring the accuracy of diagnostic system. Science, 240:1285-1293, 1986. 
[48] J. A. Swets, R. M. Dawes, and J. Monahan. Better decisions through science. Scientific American, 283(4):82-87, October 2000.

[49] R. D. Thompson and A. P. Basu. Asymmetric loss functions for estimating system reliability. Bayesian Analysis in Statistics and Econometrics, John Wiley \& Sons, pages $471-482,1996$.

[50] K. A. Toh, J. Kim, and S. Lee. Maximizing area under ROC curve for biometric scores fusion. Pattern Recognition, 41(11):3373 - 3392, 2008.

[51] L. Torgo. Regression error characteristic surfaces. In Proceedings of the eleventh ACM SIGKDD international conference on Knowledge discovery in data mining, pages 697-702. ACM, 2005.

[52] P. Turney. Types of cost in inductive concept learning. Canada National Research Council Publications Archive, 2000.

[53] H. R. Varian. A bayesian approach to real estate assessment. Studies in Bayesian econometrics and statistics in honor of Leonard J. Savage, pages 195-208, 1975.

[54] A. Zellner. Bayesian estimation and prediction using asymmetric loss functions. Journal of the American Statistical Association, pages 446-451, 1986.

[55] H. Zhao, A. P. Sinha, and G. Bansal. An extended tuning method for cost-sensitive regression and forecasting. Decision Support Systems, 2011.

\section{Appendix. Proof of the connection between $A O C$ and error variance}

We include the proof of theorem 7:

$$
A O C=\frac{\sigma^{2} n^{2}}{2}
$$

Proof. We start with an error vector $\mathbf{e}$ of length $n$, which we assume is sorted in decreasing order, as in algorithm 1. We use a different notation for the points on the RROC curve. Instead of using $n+2$ points, we will just ignore the two extremes (which do not contribute to the area for finite cases) and we will just work with $n$ points, denoted by $p_{1}, \ldots, p_{n}$. The components of each point are $p_{i}=\left(o_{i}, u_{i}\right)$. Note that $o_{i}=R R O C X_{i+1}$ using the notation in algorithm 1 and $u_{i}=R R O C Y_{i+1}$. We will also introduce the error differences $d_{i}=e_{i}-e_{i+1}$, which are defined from $i=1$ to $i=n-1$. Note that $d_{i} \geq 0$ since the error vector $\mathbf{e}$ is in decreasing order. It is easy to see that $o_{i}=\sum_{j=1}^{i-1} j \cdot d_{j}$ and $u_{i}=-\sum_{j=i}^{n-1}(n-j) \cdot d_{j}$. According to this notation:

$$
A O C=-\sum_{i=1}^{n-1} \frac{u_{i}+u_{i+1}}{2}\left(o_{i+1}-o_{i}\right)
$$

In order to prove this theorem, we will proceed by induction.

\section{Base case}

The base case will consider any error vector of size $n=2$. In this case, we only have 
two points $p_{1}=\left(0,-d_{1}\right)$ and $p_{2}=\left(d_{1}, 0\right)$. From here,

$$
\begin{aligned}
A O C & =-\sum_{i=1}^{1} \frac{u_{i}+u_{i+1}}{2}\left(o_{i+1}-o_{i}\right)=-\frac{-d_{1}+0}{2}\left(d_{1}-0\right)=\frac{d_{1}^{2}}{2}=\frac{\left(e_{2}-e_{1}\right)^{2}}{2} \\
& =\frac{\left(e_{2}-\mu+\mu-e_{1}\right)^{2}}{2}=\frac{\left(e_{2}-\mu\right)^{2}+\left(\mu-e_{1}\right)^{2}+2\left(e_{2}-\mu\right)\left(\mu-e_{1}\right)}{2} \\
& =\frac{2\left(e_{2}-\mu\right)^{2}+2\left(\mu-e_{1}\right)^{2}}{2}=\frac{4 \sigma^{2}}{2}=\frac{\sigma^{2} n^{2}}{2}
\end{aligned}
$$

\section{Inductive step}

We assume that the following expression holds for any dataset of size $n$ :

$$
A O C=\frac{\sigma^{2} n^{2}}{2}
$$

Without loss of generality, we consider that the case for $n+1$ is constructed by adding example $e_{n+1}$, assumed to be lower than the other examples $e_{1}, e_{2}, \ldots, e_{n}$ coming from the $n$ case. Consequently, the error vector for the case $n+1$ is $e_{1}, e_{2}, \ldots, e_{n}, e_{n+1}$. The difference vector is also an extension for $n+1$, denoted by $d_{1}, d_{2}, \ldots, d_{n}$. Note that since we assume that eq. (.1) holds for any dataset of $n$ examples, we can choose the order of examples that we prefer in order to build any case with $n+1$ examples.

The $A O C$ for the $n$ case is given by:

$$
A O C=-\sum_{i=1}^{n-1} \frac{u_{i}+u_{i+1}}{2}\left(o_{i+1}-o_{i}\right)
$$

The $A O C$ for the $n+1$ case is given by

$$
\widetilde{A O C}=-\sum_{i=1}^{n} \frac{\widetilde{u}_{i}+\widetilde{u}_{i+1}}{2}\left(\widetilde{o}_{i+1}-\widetilde{o}_{i}\right)
$$

We will use a wide tilde to denote the $\widetilde{A O C}, \widetilde{\sigma}, \widetilde{\mu}$, etc., for the $n+1$ case. The first thing we can see is that $\widetilde{u}_{1}=u_{1}-d_{1}-d_{2}-\cdots-d_{n}, \widetilde{u}_{2}=u_{2}-d_{2}-\cdots-d_{n}$, etc. We use these latter expressions on eq. (.2):

$$
\widetilde{A O C}=-\sum_{i=1}^{n} \frac{u_{i}+\left\{-\sum_{j=i}^{n} d_{j}\right\}+u_{i+1}+\left\{-\sum_{j=i+1}^{n} d_{j}\right\}}{2}\left(\widetilde{o}_{i+1}-\widetilde{o}_{i}\right)
$$

The second thing we realise is that $o_{i}$ and $\widetilde{o}_{i}$ are equal for $i=1 \ldots n$. From here, we can calculate the delta between $n+1$ and $n$ as follows:

$$
\triangle A O C \triangleq \widetilde{A O C}-A O C=-\sum_{i=1}^{n} \frac{\left\{-\sum_{j=i}^{n} d_{j}\right\}+\left\{-\sum_{j=i+1}^{n} d_{j}\right\}}{2}\left(\widetilde{o}_{i+1}-\widetilde{o}_{i}\right)
$$


But we have that $\widetilde{o}_{i+1}-\widetilde{o}_{i}=i \cdot d_{i}$. So, we rewrite:

$$
\begin{aligned}
\triangle A O C & =-\sum_{i=1}^{n} \frac{\left\{-\sum_{j=i}^{n} d_{j}\right\}+\left\{-\sum_{j=i+1}^{n} d_{j}\right\}}{2}\left(i \cdot d_{i}\right) \\
& =\sum_{i=1}^{n} \frac{\left(d_{i}+2 \sum_{j=i+1}^{n} d_{j}\right)\left(i \cdot d_{i}\right)}{2}=\sum_{i=1}^{n} \frac{i \cdot d_{i}^{2}+2 \sum_{j=i+1}^{n} i \cdot d_{i} d_{j}}{2}
\end{aligned}
$$

Using the expression of the square of a sum: $\left(\sum_{i} a_{i}\right)^{2}=\sum_{i} a_{i}^{2}+2 \sum_{i<j} a_{i} a_{j}$, and joining/distributing terms, we see that the above expression can be rewritten as:

$$
\begin{aligned}
\triangle A O C & =\sum_{i=1}^{n} \frac{\left\{\sum_{j=i}^{n} d_{i}\right\}^{2}}{2}=\sum_{i=1}^{n} \frac{\left(e_{n+1}-e_{i}\right)^{2}}{2}=\sum_{i=1}^{n} \frac{e_{n+1}^{2}-2 e_{n+1} e_{i}+e_{i}^{2}}{2} \\
& =\frac{1}{2}\left(n \cdot e_{n+1}^{2}-2 e_{n+1} \sum_{i=1}^{n} e_{i}+\sum_{i=1}^{n} e_{i}^{2}\right)=\frac{1}{2}\left\{n \cdot e_{n+1}^{2}-2 e_{n+1} n \cdot \mu+n\left(\sigma^{2}+\mu^{2}\right)\right\} \\
& =\frac{n}{2}\left(e_{n+1}^{2}-2 e_{n+1} \mu+\sigma^{2}+\mu^{2}\right)=\frac{n}{2}\left\{\left(e_{n+1}-\mu\right)^{2}+\sigma^{2}\right\}
\end{aligned}
$$

From here, we can now write:

$$
\widetilde{A O C}=A O C+\triangle A O C=A O C+\frac{n}{2}\left(\left(e_{n+1}-\mu\right)^{2}+\sigma^{2}\right)
$$

From the induction step (eq. .1), we have:

$$
\begin{aligned}
\widetilde{A O C} & =\frac{\sigma^{2} n^{2}}{2}+\frac{n}{2}\left(\left(e_{n+1}-\mu\right)^{2}+\sigma^{2}\right)=\frac{n}{2}\left(\sigma^{2} n+\left(e_{n+1}-\mu\right)^{2}+\sigma^{2}\right) \\
& =\frac{n}{2}\left(\sigma^{2}(n+1)+\left(e_{n+1}-\mu\right)^{2}\right)=\frac{n}{2}\left\{\left(\frac{\sum_{i=1}^{n} e_{i}^{2}}{n}-\mu^{2}\right)(n+1)+\left(e_{n+1}-\mu\right)^{2}\right\} \\
& =\frac{1}{2}\left\{\left(\sum_{i=1}^{n} e_{i}^{2}-n \mu^{2}\right)(n+1)+n \cdot\left(e_{n+1}-\mu\right)^{2}\right\} \\
& =\frac{1}{2}\left\{\left(\sum_{i=1}^{n+1} e_{i}^{2}\right)(n+1)-\left(n \mu^{2}\right)(n)-e_{n+1}^{2}-2 n \cdot e_{n+1} \mu\right\} \\
& =\frac{1}{2}\left\{\left(\sum_{i=1}^{n+1} e_{i}^{2}\right)(n+1)-\left(n \mu+e_{n+1}\right)^{2}\right\}=\frac{1}{2}\left\{\left(\sum_{i=1}^{n+1} e_{i}^{2}\right)(n+1)-((n+1) \widetilde{\mu})^{2}\right\} \\
& =\frac{1}{2}(n+1)^{2}\left(\frac{\sum_{i=1}^{n+1} e_{i}^{2}}{n+1}-\widetilde{\mu}^{2}\right)=\frac{1}{2}(n+1)^{2}\left(\widetilde{\sigma}^{2}\right)=\frac{\widetilde{\sigma}^{2}(n+1)^{2}}{2}
\end{aligned}
$$

This last expression completes the induction step and the proof. 